\title{
Bounding the Inefficiency of the Multiclass, Multicriteria C-Logit Stochastic User Equilibrium in a Transportation Network
}

\author{
Lekai Yuan $\mathbb{D}^{1},{ }^{1}$ Xi Zhang $\mathbb{D}^{1},{ }^{1}$ and Chaofeng Shi $\mathbb{D}^{2}$ \\ ${ }^{1}$ College of Traffic and Transportation, Chongqing Jiaotong University, Chongqing 400074, China \\ ${ }^{2}$ School of Economics and Management, Chongqing Jiaotong University, Chongqing 400074, China
}

Correspondence should be addressed to Xi Zhang; xzhang@cqjtu.edu.cn

Received 28 August 2021; Revised 24 November 2021; Accepted 27 November 2021; Published 27 December 2021

Academic Editor: Inés P. Mariño

Copyright (c) 2021 Lekai Yuan et al. This is an open access article distributed under the Creative Commons Attribution License, which permits unrestricted use, distribution, and reproduction in any medium, provided the original work is properly cited.

We derive the exact inefficiency upper bounds of the multiclass C-Logit stochastic user equilibrium (CL-SUE) in a transportation network. All travelers are classified on the basis of different values of time (VOT) into M classes. The multiclass CL-SUE model gives a more realistic path choice probability in comparison with the logit-based stochastic user equilibrium model by considering the overlapping effects between paths. To find efficiency loss upper bounds of the multiclass CL-SUE, two equivalent variational inequalities for the multiclass CL-SUE model, i.e., time-based variational inequality (VI) and monetary-based VI, are formulated. We give four different methods to define the inefficiency of the multiclass CL-SUE, i.e., to compare multiclass CL-SUE with multiclass system optimum, or to compare multiclass CL-SUE with multiclass C-Logit stochastic system optimum (CL-SSO), under the time-based criterion and the monetary-based criterion, respectively. We further investigate the effects of various parameters which include the degree of path overlapping (the commonality factor), the network complexity, degree of traffic congestion, the VOT of user classes, the network familiarity, and the total demand on the inefficiency bounds.

\section{Introduction}

Concerning the path choice behavior in networks, Wardrop proposed two basic principles: one is the user equilibrium (UE) and the other is the system optimum (SO) [1]. The UE principle shows that all used paths have minimum and equal travel cost (or time), and all unused paths have higher or equal travel cost (or time). In addition, the UE principle assumes that every traveler has a full understanding of the network information and chooses the travel path accordingly to minimize their own travel cost. Later, the researchers have extended the UE model in different aspects, such as boundedly rational UE [2-4], fuzzy UE [5-7], and prospect-based UE [8-10]. The concept of the boundedly rational user equilibrium (BRUE) was proposed in the 1980s. Lou et al. [3] investigated congestion pricing strategies in static networks with boundedly rational route choice behavior. Xuan et al. [4] studied mathematical formulation and solution sets of BRUE. Miralinaghi et al. [7] proposed an alternative approach for a traffic assignment problem that further extends the applications of the fuzzy theory in route choice behavior and network traffic modelling. Xu et al. [10] proposed a conjecture on travelers' determination of reference points and encapsulates it into the prospect-based user equilibrium conditions. However, in practice, we cannot always assume that this assumption is true. More realistically, travelers who choose the same route may have different perceived travel times due to all kinds of unmeasured factors. Suppose that the perceived travel times are considered as independent and identical distributed (IID) Gumbel random variables [11], travelers' path choices for minimizing their perceived travel time will lead to the stochastic user equilibrium (SUE) state [12]. Concerning the path choice problem in the network, two disadvantages of the logit-based SUE model are as follows: (1) it cannot explain overlapping between paths and (2) it cannot explain the perception variance of travels of different lengths $[13,14]$.

To alleviate the above two disadvantages, some extended logit-based SUE models have been proposed in recent two 
decades, such as C-Logit SUE (CL-SUE) model [13-16], generalized nested logit SUE (GNL-SUE) model [17], crossnested logit SUE (CNL-SUE) model $[18,19]$, and paired combinatorial logit SUE (PCL-SUE) model [20]. Other significant theoretical achievements in [21,22] have also made a contribution to overcoming the disadvantages of the logit-based SUE model. CL-SUE model proposed by Cascetta et al. [13] can solve the overlapping problem by using a commonality factor reflecting path overlapping to modify the systematic part of the utility function. Zhou et al. [14] further provided equivalent variational inequality (VI) and mathematical programming (MP) formulations for the CLSUE model.

The CL-SUE model can overcome the disadvantages of the logit-based SUE model by considering the overlapping effects between paths and give a more realistic path choice probability. The SO state has the minimum inefficiency loss by definition. Therefore, the CL-SUE model is still inefficient compared with the SO model due to the users' selfish routing. Recently, quantifying and bounding the inefficiency of the equilibrium assignment in the transportation environment has aroused great research interest, while less attention has been paid to multiclass CLSUE. Koutsoupias and Papadimitriou first gave the concept of "price of anarchy (POA)" [23]. After that, the POA was first used in the traffic network by Roughgarden and Tardos [24]. In the next few years, other great theoretical achievements of POA proposed by Roughgarden [25-27] have greatly promoted the development of inefficiency in the transportation network. In recent years, the above works have been extensively investigated in many aspects [28-36]. Guo et al. [37] gave two efficiency loss bounds of the SUE against SO and stochastic system optimum (SSO) in a stochastic circumstance, respectively. Considering a transportation network with multiple classes of users, $\mathrm{Yu}$ et al. [38] studied the inefficiency of the multiclass SUE, which is the extension of research achievements in [37]. Based on the above main work, some researchers have also studied the inefficiency of the extended logit-based SUE. Yong et al. provided several efficiency loss upper bounds of the CL-SUE by considering the overlapping effects between paths [39], which is also the extension of research achievements in [37]. Zeng and Wang initially explored inefficiency upper bound of CNL-SUE in the taxed stochastic transportation network and further investigated inefficiency upper bounds for the low-degree travel time function [40]. For a transportation network with heterogeneous users who have different values of time (VOT), the network optimization usually has two objectives, i.e., minimizing total system travel time (TSTT) and minimizing total system travel cost (TSTC). Guo and Yang further measured the system optimal performance difference by the two different criteria, i.e., time-based criteria and monetary-based criteria [41]. Yu and Wang [42] derived the accurate inefficiency bounds of multiclass UE with elastic travel demand by making full use of equivalent VI under time-based criterion and cost-based criterion, respectively. Han and Yang [43] have given several bounds for the inefficiency of the multiclass traffic equilibrium assignment problem in the tolled network under the two different criteria, respectively. Huang et al. [44] further discussed the efficiency loss of the SUE where all travelers are classified into two main categories, one equipped with advanced traveler information systems (ATIS) and another unequipped. Yu et al. studied the inefficiency of the mixed equilibrium with heterogeneous users [45-47].

Different from the existing studies, this study extends the logit-based SUE to the multiclass CL-SUE. The purpose of our study was to derive four efficiency loss upper bounds of the multiclass CL-SUE. The two corresponding models, i.e., multiclass $\mathrm{SO}$ model and multiclass C-Logit stochastic system optimum (CL-SSO) model, should be mentioned before exploring the efficiency loss of the multiclass CL-SUE. The multiclass SO model that minimizes the TSTT has been widely used in the literature. In this article, the multiclass CL-SSO model that minimizes the total perceived travel time (TPTT) is established based on the work in $[39,48,49]$. Therefore, there are four kinds of ways to define the efficiency loss of the multiclass CL-SUE, i.e., comparing multiclass CL-SUE with multiclass SO, or comparing multiclass CL-SUE with multiclass CL-SSO, under the time-based criterion and the monetary-based criterion, respectively. The highlights of our research focus on these four situations. In recent years, the effects of various parameters (e.g., degree of traffic congestion and total traffic demand of the network) on the inefficiency upper bound has been widely studied, while paying less attention to commonality factor reflecting path overlapping. This paper studies the effects of commonality factor reflecting path overlapping on the efficiency loss of the multiclass CL-SUE. The result shows that the commonality factor reflecting path overlapping has a significant impact on the inefficiency upper bound.

The rest of this article is organized below. In Section 2, we have a brief review of multiclass SUE and CL-SUE models, and then formulate the equivalent VI formulations of the multiclass CL-SUE model under time-based criterion and monetary-based criterion, respectively. In Sections 3 and 4 , we derive four inefficiency upper bounds by using the equivalent VI formulations of the multiclass CL-SUE. Section 5 discusses the effects of various parameters on the efficiency loss bounds. In Section 6, we summarize the main research findings of this paper in tabular form. We give a numerical example to illustrate our conclusions in Section 7. Finally, Section 8 provides some conclusions and analyses the further research directions.

\section{Review of Multiclass SUE and CL-SUE Models}

2.1. Multiclass SUE Model. Let $G(N, A)$ be a directed transportation network defined by a set $N$ of nodes and a set $A$ of directed links. All travelers are classified in terms of different VOT into $M$ classes. Table 1 provides the notations used in the paper:

According to Table 1, the following relationships and constraints hold: 
TABle 1: Notations.

\begin{tabular}{|c|c|}
\hline Notation & Description \\
\hline W & The set of origin-destination (OD) pairs \\
\hline$R_{w}$ & The set of all feasible paths connecting OD pair $w \in W$ \\
\hline$\beta_{m}^{\omega}\left(\beta_{m}>0\right)$ & The average VOT for users of class $m$ \\
\hline$d_{w}^{m}$ & The travel demand of user class $m$ between OD pair $w \in W$ \\
\hline$f_{r w}^{m}$ & The flow of user class $m$ on path $r \in R_{w}, w \in W$ \\
\hline$f$ & Path flow vector, $f=\left(f_{r w}^{m}, r \in R_{w}, w \in W, m=1,2, \ldots, M\right)^{T}$ \\
\hline$v_{a}^{m}$ & The flow of user class $m$ on link $a \in A$ \\
\hline$v_{a}$ & The total flow on link $a \in A$ \\
\hline$v$ & Link flow vector, $v=\left(v_{a}^{m}, a \in A, m=1,2, \ldots, M\right)^{T}$ \\
\hline$\delta_{a r}^{w}$ & The link-path incidence indicator, which is equal to 1 if path $r \in R_{w}$ uses link $a \in A$, and 0 otherwise \\
\hline$\Omega_{f}$ & Set of feasible path flow vectors, $\Omega_{f}=\left\{f \mid f_{r w}^{m} \geq 0, \sum_{r \in R_{w}} f_{r w}^{m}=d_{w}^{m}, \forall r \in R_{w}, \forall w \in W, m=1,2, \ldots, M\right\}$ \\
\hline$\Omega_{v}$ & Set of feasible link flow vectors, $\Omega_{v}=\left\{v \mid f \in \Omega_{f}, v_{a}^{m}=\sum_{w \in W} \sum_{r \in R_{w}} f_{r w}^{m} \delta_{a r}^{w}, v_{a}=\sum_{m=1}^{M} v_{a}^{m}, \forall a \in A, m=1,2, \ldots, M\right\}$ \\
\hline$t_{a}\left(v_{a}\right)$ & $\begin{array}{l}\text { The travel time function on link } a \in A \text {, which is supposed to be separable, convex, differentiable, and monotonically increasing } \\
\text { with } v_{a}\end{array}$ \\
\hline $\begin{array}{l}c_{a}^{m}\left(v_{a}\right) \\
c_{r w}^{m}\end{array}$ & $\begin{array}{l}\text { The actual travel time of user class } m \text { on link } a \in A \text {, which is assumed to be a monotonically increasing function of } v_{a} \\
\text { The actual travel time of user class } m \text { on path } r \in R_{w}, w \in W\end{array}$ \\
\hline
\end{tabular}

$$
\begin{aligned}
v_{a}^{m} & =\sum_{w \in W} \sum_{r \in R_{w}} f_{r w}^{m} \delta_{a r}^{w}, \forall a \in A, m=1,2, \cdots, M, \\
v_{a} & =\sum_{m=1}^{M} v_{a}^{m}, \forall a \in A, m=1,2, \cdots, M, \\
\sum_{r \in R_{w}} f_{r w}^{m} & =d_{w}^{m}, \forall w \in W, m=1,2, \cdots, M, \\
f_{r w}^{m} & \geq 0, \forall r \in R_{w}, w \in W, m=1,2, \cdots, M, \\
c_{r w}^{m} & =\sum_{a \in A} c_{a}^{m} \delta_{a r}^{w}, \forall r \in R_{w}, w \in W, m=1,2, \cdots, M .
\end{aligned}
$$

In a multiclass SUE model, assume that all users are utilitymaximizers. Let $U_{r w}^{m}$ denote the travel utility perceived by user class $m$ on path $r \in R_{w}, w \in W$. Then, $U_{r w}^{m}$ is given by

$$
U_{r w}^{m}=-\theta C_{r w}^{m}=-\theta c_{r w}^{m}+\xi_{r w}^{m}, r \in R_{w}, w \in W, m=1,2, \cdots, M,
$$

where $C_{r w}^{m}$ is the perceived travel time of user class $m$ on path $r \in R_{w}, w \in W, \theta$ is a positive unit scaling parameter, $-\theta c_{r w}^{m}$ is the measured utility, and $\xi_{r w}^{m}$ is a random term representing the user's perception error. Let $P_{r w}^{m}$ be the probability of user class $m$ choosing path $r \in R_{w}, w \in W$, then the utility maximization theory shows that

$$
P_{r w}^{m}=\operatorname{Pr}\left(U_{r w}^{m} \geq U_{k w}^{m}, \forall k \in R_{w}\right), r \in R_{w}, w \in W .
$$

$P_{r w}^{m}$ has the two properties as follows:

$$
\begin{aligned}
0 & \leq P_{r w}^{m} \leq 1, r \in R_{w}, w \in W, m=1,2, \cdots, M, \\
\sum_{r \in R_{w}} P_{r w}^{m} & =1, w \in W, m=1,2, \cdots, M .
\end{aligned}
$$

All random terms $\xi_{r w}^{m}$ in (2) are supposed to be IID Gumbel random variables. Then, the path choice probability for user class $m$ is given by

$$
P_{r w}^{m}=\frac{\exp \left(-\theta c_{r w}^{m}\right)}{\sum_{l \in R_{w}} \exp \left(-\theta c_{l w}^{m}\right)}, r \in R_{w}, w \in W, m=1,2, \cdots, M
$$

and $f_{r w}^{m}$ can be obtained by

$$
f_{r w}^{m}=d_{w}^{m} P_{r w}^{m}, r \in R_{w}, w \in W, m=1,2, \cdots, M .
$$

Similar to that done in Fisk [12], the multiclass logitbased SUE model can be formulated as an equivalent MP problem below.

$$
\min _{f \in \Omega_{f}} Z(f)=\sum_{a \in A} \int_{0}^{v_{a}} t_{a}(\omega) d \omega+\frac{1}{\theta} \sum_{w \in W} \sum_{r \in R_{w}} \sum_{m=1}^{M} f_{r w}^{m} \ln f_{r w}^{m} .
$$

The equivalent VI of problem (7) is presented in the lemma below.

Lemma 1. If the link time function, $t_{a}\left(v_{a}\right), a \in A$ is differentiable, convex, separable, and monotonically increasing with link flow $v_{a}$, a multiclass logit-based SUE model with fixed OD demand is equivalent to the following VI, i.e., find $\vec{f} \in \Omega_{f}$, such that

$$
\sum_{w \in W} \sum_{r \in R_{w}} \sum_{m=1}^{M}\left(c_{r w}^{m}(\vec{f})+\frac{1}{\theta} \ln \vec{f}_{r w}^{m}\right)\left(f_{r w}^{m}-\vec{f}_{r w}^{m}\right) \geq 0, \forall f \in \Omega_{f} .
$$

The disadvantages of the multiclass SUE model have been found by some researchers. One of the disadvantages is that it cannot explain the overlapping effects between different paths, which means that the unrealistic path choice probability is given.

2.2. Multiclass CL-SUE Model. Cascetta et al. [13] proposed a CL-SUE model, which can overcome the disadvantages of the logit-based SUE by using a commonality factor reflecting path 
overlapping to modify the systematic part of the utility function. Similar to the work of Cascetta et al. [13], the multiclass CL-SUE model can be established. Then, the path choice probability of the multiclass CL-SUE model is provided by

$$
P_{r w}^{m}=\frac{\exp \left(-\theta\left(c_{r w}^{m}+c f_{r w}\right)\right)}{\sum_{l \in R_{w}} \exp \left(-\theta\left(c_{l w}^{m}+c f_{r w}\right)\right)}, r \in R_{w}, w \in W, m=1,2, \cdots, M,
$$

where $c f_{r w}$ is a commonality factor of path $r \in R_{w}, w \in W$. In this paper, the form of the commonality factor [13] is used as follows:

$$
c f_{r w}=\beta_{0} \ln \sum_{l \in R_{w}}\left(\frac{L_{r l}^{w}}{\sqrt{L_{r}^{w} \cdot L_{l}^{w}}}\right)^{\gamma_{0}}, \forall r \in R_{w}, w \in W,
$$

where $\beta_{0}$ and $\gamma_{0}$ are the parameters; $L_{r l}^{w}$ denotes the common length of paths $r$ and $l$ between the OD pair $w \in W$; and $L_{r}^{w}$ and $L_{l}^{w}$ denote the lengths of paths $r$ and $l$ between the OD pair $w \in W$, respectively. The length can be regarded as the free-flow travel time [14]. And $f_{r w}^{m}$ is provided by

$$
f_{r w}^{m}=d_{w}^{m} P_{r w}^{m}, r \in R_{w}, w \in W, m=1,2, \cdots, M .
$$

Similar to that done in Zhou et al. [14], the multiclass CL-SUE model can be formulated as an equivalent MP problem below.

$$
\begin{aligned}
\min _{f \in \Omega_{f}} Z(f)= & \sum_{a \in A} \int_{0}^{v_{a}} t_{a}(\omega) d \omega+\frac{1}{\theta} \sum_{w \in W} \sum_{r \in R_{w}} \sum_{m=1}^{M} f_{r w}^{m} \ln f_{r w}^{m} \\
& +\sum_{w \in W} \sum_{r \in R_{w}} \sum_{m=1}^{M} f_{r w}^{m} c f_{r w} .
\end{aligned}
$$

To find efficiency loss upper bounds of the multiclass CLSUE, the equivalent VI of problem (12) is presented in the lemma below.

Lemma 2. If the link time function, $t_{a}\left(v_{a}\right), a \in A$ is differentiable, convex, separable, and monotonically increasing with link flow $v_{a}$, a multiclass CL-SUE model with fixed OD demand is equivalent to the following VI, i.e., find $\bar{f} \in \Omega_{f}$, such that

$$
\sum_{w \in W} \sum_{r \in R_{w}} \sum_{m=1}^{M}\left(c_{r w}^{m}(\bar{f})+\frac{1}{\theta} \ln \bar{f}_{r w}^{m}+c f_{r w}\right)\left(f_{r w}^{m}-\bar{f}_{r w}^{m}\right) \geq 0, \forall f \in \Omega_{f} .
$$

Proof of Lemma 2. Since $t_{a}\left(v_{a}\right)$ is monotonically increasing and $\Omega_{f}$ is compact, the objective function (12) is strictly convex. Hence, the path flow solution of the problem (12) is unique. The necessary and sufficient condition, which can guarantee that $\bar{f} \in \Omega_{f}$ is the unique optimal solution of problem (12), is provided as follows:

$$
\left[\nabla_{f} Z(\bar{f})\right]^{T}(f-\bar{f}) \geq 0 \text {, for any } f \in \Omega_{f} .
$$

Using $\quad v_{a}=\sum_{m=1}^{M} v_{a}^{m}=\sum_{w \in W} \sum_{r \in R_{w}} \sum_{m \in M} f_{r w}^{m} \delta_{a r}^{w} \quad$ and substituting

$$
\begin{array}{r}
{\left[\nabla_{f} Z(\bar{f})\right]^{T}=\left[\cdots, \frac{1}{\theta}+\frac{1}{\theta} \ln \bar{f}_{r w}^{m}+\sum_{a \in A} t_{a}\left(\bar{v}_{a}\right) \delta_{a r}^{w}+c f_{r w}, \cdots\right]=\left[\cdots, \frac{1}{\theta}+\frac{1}{\theta} \ln \bar{f}_{r w}^{m}+c_{r w}^{m}(\bar{f})+c f_{r w}, \cdots\right] .} \\
c_{a}^{m}\left(v_{a}\right)=\beta_{m} t_{a}\left(v_{a}\right), a \in A, m=1,2, \cdots, M .
\end{array}
$$

into (14), VI (13) can be obtained according to equation $\sum_{w \in W} \sum_{r \in R_{w}} \sum_{m \in M} 1 / \theta\left(f_{r w}^{m}-\bar{f}_{r w}^{m}\right)=\sum_{w \in W} 1 / \theta\left(d_{w}-d_{w}\right)=0$.

Under the time-based criterion, $c_{a}^{m}\left(v_{a}\right)$ is given by

$$
c_{a}^{m}\left(v_{a}\right)=t_{a}\left(v_{a}\right), a \in A, m=1,2, \cdots, M .
$$

Substituting (16) and (17) into (13), respectively, and using (1), we can get the following time-based VI and monetary-based VI, respectively.

By contrast, under the monetary-based criterion, $c_{a}^{m}\left(v_{a}\right)$ can be given by

$$
\begin{array}{r}
\sum_{a \in A} t_{a}(\bar{v})\left(v_{a}-\bar{v}_{a}\right)+\frac{1}{\theta} \sum_{w \in W} \sum_{r \in R_{w}} \sum_{m=1}^{M}\left(\ln \bar{f}_{r w}^{m}+\theta c f_{r w}\right)\left(f_{r w}^{m}-\bar{f}_{r w}^{m}\right) \geq 0, \forall f \in \Omega_{f}, \\
\sum_{a \in A} \sum_{m=1}^{M} \beta_{m} t_{a}(\bar{v})\left(v_{a}^{m}-\bar{v}_{a}^{m}\right)+\frac{1}{\theta} \sum_{w \in W} \sum_{r \in R_{w}} \sum_{m=1}^{M}\left(\ln \bar{f}_{r w}^{m}+\theta c f_{r w}\right)\left(f_{r w}^{m}-\bar{f}_{r w}^{m}\right) \geq 0, \forall f \in \Omega_{f} .
\end{array}
$$




\section{Bounding the Inefficiency of the Multiclass CL-SUE Compared with the Multiclass SO}

3.1. Time Units. The multiclass SO problem in time units that minimizes the TSTT is presented as follows:

$$
\min _{v \in \Omega_{v}} \sum_{a \in A} t_{a}\left(v_{a}\right) v_{a}
$$

Let $\widehat{v} \in \Omega_{v}$ be the link flow solution of problem (19), the corresponding path flow solution is denoted by $\widehat{f} \in \Omega_{f}$. Let $\bar{v}^{t} \in \Omega_{v}$ be the link flow solution of the VI (18), the corresponding path flow solution is denoted by $\bar{f}^{t} \in \Omega_{f}$. The efficiency loss of the multiclass CL-SUE compared with the multiclass SO under time-based criterion is defined as

$$
\rho_{\mathrm{CL}-\mathrm{SUE}}^{t}=\frac{T_{\mathrm{CL}-\mathrm{SUE}}^{t}}{T_{\mathrm{SO}}^{t}}
$$

where $\quad T_{\mathrm{CL}-\mathrm{SUE}}^{t}=\sum_{w \in W} \sum_{r \in R_{w}} \sum_{m=1}^{M} c_{r w}^{m}\left(\bar{f}^{t}\right) \bar{f}_{r w}^{m, t}=\sum_{a \in A} t_{a}$ $\left(\bar{v}_{a}^{t}\right) \bar{v}_{a}^{t}$ is the TSTT at the multiclass CL-SUE and $T_{\mathrm{SO}}^{t}=$ $\sum_{a \in A} t_{a}\left(\widehat{v}_{a}\right) \widehat{v}_{a}$ is the TSTT at the multiclass SO. Clearly, $\rho_{\mathrm{CL}-\mathrm{SUE}}^{t} \geq 1$.

Setting $\bar{f}=\bar{f}^{t}$ and $f=\widehat{f}$ in VI (18), we can obtain inequality as follows:

$$
\begin{aligned}
& \sum_{a \in A} t_{a}\left(\bar{v}_{a}^{t}\right)\left(\widehat{v}_{a}-\bar{v}_{a}^{t}\right)+\frac{1}{\theta} \sum_{w \in W} \sum_{r \in R_{w}} \sum_{m=1}^{M}\left(\ln \bar{f}_{r w}^{m, t}+\theta c f_{r w}\right) \\
& \cdot\left(\hat{f}_{r w}^{m}-\bar{f}_{r w}^{m, t}\right) \geq 0 .
\end{aligned}
$$

This leads to

$$
\begin{aligned}
& T_{C L-S U E}^{t} \leq T_{S O}^{t}+\sum_{a \in A}\left(t_{a}\left(\bar{v}_{a}^{t}\right)-t_{a}\left(\widehat{v}_{a}\right)\right) \widehat{v}_{a} \\
& \quad+\frac{1}{\theta} \sum_{w \in W} \sum_{r \in R_{w}} \sum_{m=1}^{M}\left(\ln \bar{f}_{r w}^{m, t}+\theta c f_{r w}\right)\left(\hat{f}_{r w}^{m}-\bar{f}_{r w}^{m, t}\right) .
\end{aligned}
$$

Now, we begin to find the upper bound of the term $\sum_{a \in A}\left(t_{a}\left(\bar{v}_{a}^{t}\right)-t_{a}\left(\widehat{v}_{a}\right)\right) \widehat{v}_{a}$ in (22). To achieve this, we give the same way which has been used in [37]. For completeness, a brief introduction is given here. Because $t_{a}\left(v_{a}\right)$ is increasing, we have $\left(t_{a}\left(\bar{v}_{a}^{t}\right)-t_{a}\left(v_{a}\right)\right) v_{a} \leq 0$ for $v_{a} \geq \bar{v}_{a}^{t}$. Hence, we just need to pay attention to $\left(t_{a}\left(\bar{v}_{a}^{t}\right)-t_{a}\left(v_{a}\right)\right) v_{a}$ for $v_{a}<\bar{v}_{a}^{t}$. As shown in Figure 1, let $t_{a}\left(\bar{v}_{a}^{t}\right) \bar{v}_{a}^{t}$ denote the area of the large rectangle and $\left(t_{a}\left(\bar{v}_{a}^{t}\right)-t_{a}\left(v_{a}\right)\right) v_{a}$ denote the area of the blue rectangle.

Next, an upper bound of the ratio $\left(\left(t_{a}\left(\bar{v}_{a}^{t}\right)-t_{a}\left(v_{a}\right)\right) v_{a}\right) /\left(t_{a}\left(\bar{v}_{a}^{t}\right) \bar{v}_{a}^{t}\right)$ needs to be determined. To complete this, for each link time function $t_{a}=t_{a}\left(z_{a}\right)$ and nonnegative link flow $z_{a} \geq 0$, we let

$$
\gamma_{a}\left(t_{a}, z_{a}\right)=\max _{v_{a} \geq 0} \frac{\left(t_{a}\left(z_{a}\right)-t_{a}\left(v_{a}\right)\right) v_{a}}{t_{a}\left(z_{a}\right) z_{a}}, a \in A .
$$

Since $\left(t_{a}\left(z_{a}\right)-t_{a}\left(v_{a}\right)\right) v_{a} \leq t_{a}\left(z_{a}\right) v_{a}$ when $0 \leq v_{a} \leq z_{a}$ and $\left(t_{a}\left(z_{a}\right)-t_{a}\left(v_{a}\right)\right) v_{a} \leq 0$ when $v_{a}>z_{a}$, we can obtain

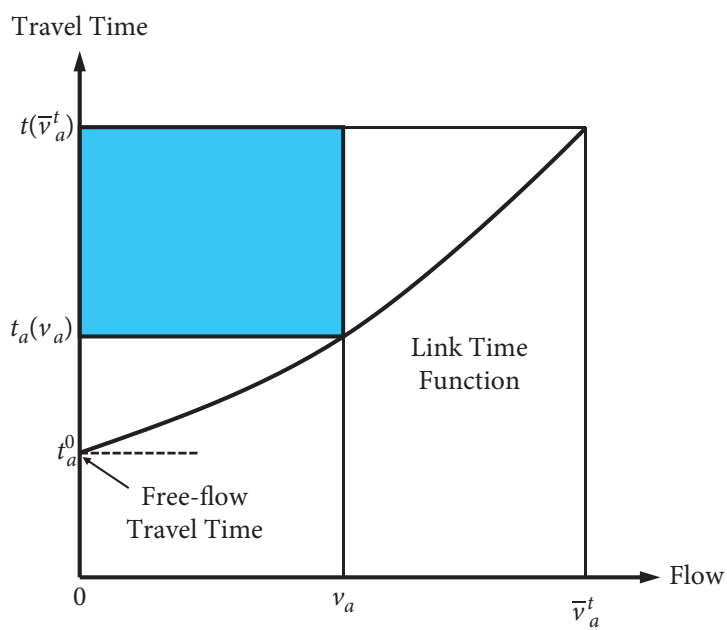

Figure 1: Geometric illustration of the definition of $\gamma(\varphi)$.

$0 \leq \gamma_{a}\left(t_{a}, z_{a}\right) \leq 1$. Let $\varphi$ denote a class of link time functions, we define

$$
\gamma(\varphi)=\max _{t_{a} \in \varphi, z_{a} \geq 0} \gamma_{a}\left(t_{a}, z_{a}\right)
$$

With definitions (23) and (24), the lemma is given as follows:

Lemma 3. Let $\bar{v}^{t}$ be link flow at the multiclass CL-SUE and $v$ denote an arbitrary nonnegative link flow. Then,

$$
\sum_{a \in A}\left(t_{a}\left(\bar{v}_{a}^{t}\right)-t_{a}\left(v_{a}\right)\right) v_{a} \leq \gamma(\varphi) T_{\mathrm{CL}-\mathrm{SUE}}^{t}
$$

Proof of Lemma 3. Setting $z_{a}=\bar{v}_{a}^{t}$ in (23) and (24), we then obtain

$$
\begin{aligned}
\sum_{a \in A}\left(t_{a}\left(\bar{v}_{a}^{t}\right)-t_{a}\left(v_{a}\right)\right) v_{a} & \leq \sum_{a \in A} \gamma_{a}\left(t_{a}, \bar{v}_{a}^{t}\right) t_{a}\left(\bar{v}_{a}^{t}\right) \bar{v}_{a}^{t} \\
& \leq \sum_{a \in A} \gamma(\varphi) t_{a}\left(\bar{v}_{a}^{t}\right) \bar{v}_{a}^{t}=\gamma(\varphi) T_{\mathrm{CL}-\mathrm{SUE}}^{t}
\end{aligned}
$$

This completes the proof.

Let $v_{a}=\widehat{v}_{a}$, we have

$\sum_{a \in A}\left(t_{a}\left(\bar{v}_{a}^{t}\right)-t_{a}\left(\widehat{v}_{a}\right)\right) \widehat{v}_{a} \leq \sum_{a \in A} \gamma(\varphi) t_{a}\left(\bar{v}_{a}^{t}\right) \bar{v}_{a}^{t}=\gamma(\varphi) T_{\mathrm{CL}-\mathrm{SUE}}^{t}$.

We now begin to seek the upper bound on the third term of the right-hand side of (22). To achieve this, we give the lemma which has been proved in [39] below.

Lemma 4. The maximization problem is considered as follows:

$$
\max F(x, y)=\sum_{i=1}^{n}\left(y_{i}-x_{i}\right)\left(\ln x_{i}+\theta c f_{i}\right),
$$

subject to 


$$
\begin{aligned}
& \sum_{i=1}^{n} x_{i}=d, \\
& \sum_{i=1}^{n} y_{i}=d,
\end{aligned}
$$

$$
x_{i}, y_{i} \geq 0, i=1,2, \cdots, n,
$$

where $d>0$ is a constant. $F_{\max }=k d$ is the maximum value of $F(x, y)$, where $k$ solves equation $k \exp (k+1)=\sum_{i=1, i \neq j}^{n}$ $\exp \left(\theta\left(c f_{j}-c f_{i}\right)\right)$ and $j=\arg \max i\left(c f_{i}, i=1,2, \cdots, n\right)$.

From Lemma 4, we have

$$
\sum_{r \in R_{w}}\left(\ln \bar{f}_{r w}^{m, t}+\theta c f_{r w}\right)\left(\hat{f}_{r w}^{m}-\bar{f}_{r w}^{m, t}\right) \leq k_{w}^{\mathrm{CL}-\mathrm{SUE}} d_{w}^{m}
$$

where $k_{w}^{\mathrm{CL}-\mathrm{SUE}}$ solves the equation $k \exp (k+1)=\sum_{r=1, r \neq j}^{\left|R_{w}\right|}$ $\exp \left(\theta\left(c f_{j w}-c f_{r w}\right)\right) \quad$ with $j=\arg \max r\left(c f_{r w}, r \in R_{w}\right)$, $w \in W$, and $\left|R_{w}\right|$ is the number of paths connecting OD pair $w \in W$. Substituting (27) and (30) into (22), it yields

$$
T_{\mathrm{CL}-\mathrm{SUE}}^{t} \leq T_{\mathrm{SO}}^{t}+\gamma(\varphi) T_{\mathrm{CL}-\mathrm{SUE}}^{t}+\frac{1}{\theta} \sum_{w \in W} \sum_{m=1}^{M} k_{w}^{\mathrm{CL}-\mathrm{SUE}} d_{w}^{m} .
$$

Let $D=\sum_{w \in W} \sum_{m=1}^{M} d_{w}^{m}$ denote the total traffic demand, $\bar{k}=\sum_{w \in W} \sum_{m=1}^{M}\left(d_{w}^{m} / D\right) k_{w}^{C L-S U E}$ be the average of $k_{w}^{\mathrm{CL}-\mathrm{SUE}}$, and $w \in W$. Then, we can rewrite (31) as

$$
T_{\mathrm{CL}-\mathrm{SUE}}^{t} \leq T_{\mathrm{SO}}^{t}+\gamma(\varphi) T_{\mathrm{CL}-\mathrm{SUE}}^{t}+\frac{1}{\theta} \bar{k} D
$$

In addition, we further define $\bar{c}=T_{\mathrm{SO}}^{t} / D$ as the actual average travel time of all travelers at multiclass SO. Then, the theorem is presented as follows:

Theorem 1. Let $\varphi$ denote a class of differentiable, separable, convex, and monotonically increasing link time functions $t_{a}\left(v_{a}\right)$. Let $T_{C L-S U E}^{t}$ be the TSTT at the multiclass CL-SUE and $T_{S O}^{t}$ be the TSTT at the multiclass SO under time-based criterion. Then,

$$
\rho_{\mathrm{CL}-\mathrm{SUE}}^{t}=\frac{T_{\mathrm{CL}-\mathrm{SUE}}^{t}}{T_{\mathrm{SO}}^{t}} \leq\left(\frac{1}{1-\gamma(\varphi)}\right)\left(1+\frac{\bar{k}}{\theta \bar{c}}\right) .
$$

Furthermore, we can have the following corollary by comparing the efficiency loss upper bounds of the multiclass CL-SUE and the multiclass SUE.

Corollary 1. In a transportation network, the efficiency loss upper bound against the multiclass SO by the multiclass CLSUE under time-based criterion is not less than that by the multiclass SUE.

Proof of Corollary 1. Let $\vec{v} \in \Omega_{v}$ be link flow solution of the VI (8), the corresponding path flow solution is denoted by $\vec{f} \in \Omega_{f} . \quad T_{\mathrm{SUE}}^{t}=\sum_{w \in W} \sum_{r \in R_{w}} \sum_{m=1}^{M} c_{r w}^{m}(\vec{f}) \vec{f}_{r w}^{m}=\sum_{a \in A} t_{a}$ $\left(\vec{v}_{a}\right) \vec{v}_{a}$ is the TSTT at the multiclass SUE. The inefficiency upper bound of the multiclass SUE against the multiclass SO under time-based criterion was given by $\mathrm{Yu}$ et al. [38], i.e.,

$$
\rho_{\mathrm{SUE}}^{t}=\frac{T_{\mathrm{SUE}}^{t}}{T_{\mathrm{SO}}^{t}} \leq\left(\frac{1}{1-\gamma(\varphi)}\right)\left(1+\frac{\vec{k}}{\theta \bar{c}}\right)
$$

where $\vec{k}=\sum_{w \in W} \sum_{m=1}^{M}\left(d_{w}^{m} / D\right) k_{w}^{\mathrm{SUE}}$ is the average of $k_{w}^{\mathrm{SUE}}$, $w \in W$, and $k_{w}^{\mathrm{SUE}}$ solves the equation $k \exp (k+1)=\left|R_{w}\right|$ $-1, w \in W$.

We can see that the difference between (33) and $\underset{k}{(34)}$ is only dependent upon the difference between $\bar{k}$ and $\vec{k}$, or $k_{w}^{\mathrm{CL}-\mathrm{SUE}}$ and $k_{w}^{\mathrm{SUE}} \cdot k_{w}^{\mathrm{CL}-\mathrm{SUE}}$ is the solution of $k \exp (k+1)=$ $\sum_{r=1, r \neq j}^{\left|R_{w}\right|} \exp \left(\theta\left(c f_{j w}-c f_{r w}\right)\right)$ with $j=\arg \max r\left(c f_{r w}, r \in\right.$ $\left.R_{w}\right), w \in W$. We always have $\sum_{r=1, r \neq j}^{\left|R_{w}\right|} \exp \left(\theta\left(c f_{j w}-c f_{r w}\right)\right) \geq$ $\left|R_{w}\right|-1$, when $j=\arg \max r\left(c f_{r w}, r \in R_{w}\right), w \in W$. Since $f(k)=k \exp (k+1)$ is a nondecreasing function of $k$, we can obtain $k_{w}^{\mathrm{CL}-\mathrm{SUE}} \geq k_{w}^{\mathrm{SUE}}$, which implies that $\bar{k} \geq \vec{k}$. Hence, the upper bound (33) is not less than the upper bound (34). This completes the proof.

The above analyses show that the commonality factor reflecting path overlapping has a significant impact on the inefficiency upper bound (33). Therefore, it is meaningful to study the efficiency loss of the multiclass CL-SUE model, which provides a more realistic upper bound than the multiclass SUE model by considering the path overlapping problem.

3.2. Monetary Units. The multiclass SO problem in monetary units that minimizes the TSTC is presented as follows:

$$
\min _{v \in \Omega_{v}} \sum_{a \in A} \sum_{m=1}^{M} \beta_{m} t_{a}\left(v_{a}\right) v_{a}^{m} .
$$

Let $\widetilde{v} \in \Omega_{v}$ be link flow solution of problem (35), the corresponding path flow solution is denoted by $\widetilde{f} \in \Omega_{f}$. Let $\bar{v}^{c} \in \Omega_{v}$ be link flow solution of the VI (18), the corresponding path flow solution is denoted by $\bar{f}^{c} \in \Omega_{f}$. The efficiency loss of the multiclass CL-SUE compared with the multiclass SO under monetary-based criterion is defined as

$$
\rho_{\mathrm{CL}-\mathrm{SUE}}^{c}=\frac{T_{\mathrm{CL}-\mathrm{SUE}}^{c}}{T_{\mathrm{SO}}^{c}}
$$

where $\quad T_{C L-S U E}^{c}=\sum_{w \in W} \sum_{r \in R_{w}} \sum_{m=1}^{M} c_{r w}^{m}\left(\bar{f}^{c}\right) \bar{f}_{r w}^{m, c}=\sum_{a \in A}$ $\sum_{m=1}^{M} \beta_{m} t_{a}\left(\bar{v}_{a}^{c}\right) \bar{v}_{a}^{m, c}$ is the TSTC at the multiclass CL-SUE and $T_{\text {SO }}^{c}=\sum_{a \in A} \sum_{m=1}^{M} \beta_{m} t_{a}\left(\widetilde{v}_{a}\right) \widetilde{v}_{a}^{m}$ is the TSTC at the multiclass SO. Clearly, $\rho_{\text {CL-SUE }}^{c} \geq 1$.

Setting $\bar{f}=\bar{f}^{C}$ and $f=\tilde{f}$ in VI (18), we can obtain inequality as follows: 


$$
\sum_{a \in A} \sum_{m=1}^{M} \beta_{m} t_{a}\left(\bar{v}_{a}^{c}\right)\left(\widetilde{v}_{a}^{m}-\bar{v}_{a}^{m, c}\right)+\frac{1}{\theta} \sum_{w \in W} \sum_{r \in R_{w}} \sum_{m=1}^{M}\left(\ln \bar{f}_{r w}^{m, c}+\theta c f_{r w}\right)\left(\widetilde{f}_{r w}^{m}-\bar{f}_{r w}^{m, c}\right) \geq 0
$$

This leads to

$$
T_{\mathrm{CL}-\mathrm{SUE}}^{c} \leq T_{\mathrm{SO}}^{c}+\sum_{a \in A} \sum_{m=1}^{M} \beta_{m} \widetilde{v}_{a}^{m}\left(t_{a}\left(\bar{v}_{a}^{c}\right)-t_{a}\left(\widetilde{v}_{a}\right)\right)+\frac{1}{\theta} \sum_{w \in W} \sum_{r \in R_{w}} \sum_{m=1}^{M}\left(\ln \bar{f}_{r w}^{m, c}+\theta c f_{r w}\right)\left(\widetilde{f}_{r w}^{m}-\bar{f}_{r w}^{m, c}\right) .
$$

To derive the upper bound of the term $\sum_{a \in A} \sum_{m=1}^{M} \beta_{m} \widetilde{v}_{a}^{m}\left(t_{a}\left(\bar{v}_{a}^{c}\right)-t_{a}\left(\widetilde{v}_{a}\right)\right)$ in (38), we need to define a new parameter [43]. For each link time function $t_{a}=t_{a}\left(z_{a}\right)$, nonnegative link flow $z_{a} \geq 0$, and the VOT $\beta_{m}, m=1,2, \cdots, M$, we define

$$
\gamma_{a}\left(z_{a}, t_{a}, \beta\right)=\frac{1}{\left(\sum_{m=1}^{M} \beta_{m} z_{a}^{m}\right) t_{a}\left(z_{a}\right)} \max _{v_{a}^{m} \geq 0}\left\{\left(\sum_{m=1}^{M} \beta_{m} v_{a}^{m}\right)\left(t_{a}\left(z_{a}\right)-t_{a}\left(v_{a}\right)\right)\right\} .
$$

Here, suppose that $0 / 0=0$ always holds. For guaranteeing $\gamma_{a}\left(z_{a}, t_{a}, \beta\right)<1$, we further define

$$
\bar{\gamma}(\varphi, \beta)=\sup _{t_{a} \in \varphi, a \in A_{1}} \max _{\bar{v}_{a}^{m, c} \geq 0} \gamma_{a}\left(z_{a}, t_{a}, \beta\right),
$$

where $A_{1}=\left\{a \in A \mid \gamma_{a}\left(z_{a}, t_{a}, \beta\right)<1\right\}$.

Define

$$
\widetilde{\gamma}(\varphi, \beta)=\sup _{t_{a} \in \varphi} g\left(z_{a}, t_{a}, \beta\right)
$$

And

$$
g\left(z_{a}, t_{a}, \beta\right)=\max _{v_{a}^{m} \geq 0, a \notin A_{1}} h(x)=\frac{\sum_{m=1}^{M} \beta_{m} v_{a}^{m}\left(t_{a}\left(z_{a}\right)-t_{a}\left(v_{a}\right)\right)}{\sum_{m=1}^{M} \beta_{m} v_{a}^{m} t_{a}\left(v_{a}\right)} .
$$

Hence, we can obtain $\tilde{\gamma}(\varphi, \beta)>-1$. Furthermore, $h(x) \longrightarrow-1$ when $v_{a}^{m} \longrightarrow+\infty$ and $h(x) \longrightarrow+\infty$ when $v_{a}^{m} \longrightarrow 0, m=1,2, \cdots, M$.

Let $v_{a}=\widetilde{v}_{a}$ and $z_{a}=\bar{v}_{a}^{c}$ in definition (39), we then have

$$
\sum_{m=1}^{M} \beta_{m} \widetilde{v}_{a}^{m}\left(t_{a}\left(\bar{v}_{a}^{c}\right)-t_{a}\left(\widetilde{v}_{a}\right)\right) \leq \gamma_{a}\left(\bar{v}_{a}^{c}, t_{a}, \beta\right) \sum_{m=1}^{M} \beta_{m} \bar{v}_{a}^{m, c} t_{a}\left(\bar{v}_{a}^{c}\right)
$$

Thus, we can obtain

$$
\sum_{a \in A} \sum_{m=1}^{M} \beta_{m} \widetilde{v}_{a}^{m}\left(t_{a}\left(\bar{v}_{a}^{c}\right)-t_{a}\left(\widetilde{v}_{a}\right)\right)=\sum_{a \in A_{1}} \sum_{m=1}^{M} \beta_{m} \widetilde{v}_{a}^{m}\left(t_{a}\left(\bar{v}_{a}^{c}\right)-t_{a}\left(\widetilde{v}_{a}\right)\right)+\sum_{a \notin A_{1}} \sum_{m=1}^{M} \beta_{m} \widetilde{v}_{a}^{m}\left(t_{a}\left(\bar{v}_{a}^{c}\right)-t_{a}\left(\widetilde{v}_{a}\right)\right) \leq \bar{\gamma}(\varphi, \beta) T_{\mathrm{CL}-\mathrm{SUE}}^{c}+\widetilde{\gamma}(\varphi, \beta) T_{\mathrm{SO}}^{c}
$$

From Lemma 4, the upper bound on the last term of the right-hand side of (38) can be provided by

$$
\sum_{r \in R_{w}}\left(\ln \bar{f}_{r w}^{m, c}+\theta c f_{r w}\right)\left(\tilde{f}_{r w}^{m}-\bar{f}_{r w}^{m, c}\right) \leq k_{w}^{\mathrm{CL}-\mathrm{SUE}} d_{w}^{m} .
$$

Substituting (44) and (45) into (38), it yields

$$
T_{\mathrm{CL}-\mathrm{SUE}}^{c} \leq T_{\mathrm{SO}}^{c}+\bar{\gamma}(\varphi, \beta) T_{\mathrm{CL}-\mathrm{SUE}}^{c}+\tilde{\gamma}(\varphi, \beta) T_{\mathrm{SO}}^{c}+\frac{1}{\theta} \sum_{w \in W} \sum_{m=1}^{M} k_{w}^{\mathrm{CL}-\mathrm{SUE}} d_{w}^{m} .
$$

In the same way, let $D=\sum_{w \in W} \sum_{m=1}^{M} d_{w}^{m}$ denote the total traffic demand, $\bar{k}=\sum_{w \in W} \sum_{m=1}^{M}\left(d_{w}^{m} / D\right) k_{w}^{C L-S U E}$ denote the average of $k_{w}^{\mathrm{CL}-\mathrm{SUE}}$, and $w \in W$. Then, we can rewrite (46) as

$$
T_{\mathrm{CL}-\mathrm{SUE}}^{c} \leq T_{\mathrm{SO}}^{c}+\bar{\gamma}(\varphi, \beta) T_{\mathrm{CL}-\mathrm{SUE}}^{c}+\widetilde{\gamma}(\varphi, \beta) T_{\mathrm{SO}}^{c}+\frac{1}{\theta} \bar{k} D .
$$

Furthermore, we define $\widetilde{c}=T_{S O}^{c} / D$ as the actual average travel cost of all travelers at multiclass SO. Then, the theorem is presented as follows. 
Theorem 2. Let $\varphi$ denote a class of differentiable, separable, convex, and monotonically increasing link time functions $t_{a}\left(v_{a}\right)$. Let $T_{C L-S U E}^{c}$ be the TSTC at the multiclass CL-SUE and $T_{S O}^{c}$ be the TSTC at the multiclass SO under monetary-based criterion. Then,

$$
\rho_{\mathrm{CL}-\mathrm{SUE}}^{c}=\frac{T_{\mathrm{CL}-\mathrm{SUE}}^{c}}{T_{\mathrm{SO}}^{c}} \leq \frac{1+\tilde{\gamma}(\varphi, \beta)+\bar{k} / \theta \bar{c}}{1-\bar{\gamma}(\varphi, \beta)} .
$$

\section{Bounding the Inefficiency of the Multiclass CL-SUE Compared with the Multiclass CL- SSO}

Before analyzing the inefficiency of the multiclass CL-SUE against the multiclass CL-SSO, we first give a brief introduction to the corresponding multiclass CL-SSO model. Considering commonality factor reflecting path overlapping, we establish the multiclass CL-SSO model, which is the extension of research achievements in $[39,48]$. The definition of SSO given by Maher et al. [48] states that the SSO problem is to minimize the TPTT. Therefore, the multiclass CL-SSO problem is also to minimize the TPTT. For the multiclass CL-SUE model, the TPTT in the transportation network can be provided by (the process of proof is shown in Appendix A)

$$
\begin{aligned}
F(f)= & \sum_{w \in W} \sum_{r \in R_{w}} \sum_{m=1}^{M} f_{r w}^{m}\left(c_{r w}^{m}(f)+c f_{r w}\right) \\
& +\frac{1}{\theta} \sum_{w \in W} \sum_{r \in R_{w}} \sum_{m=1}^{M} f_{r w}^{m} \ln f_{r w}^{m}-\frac{1}{\theta} \sum_{w \in W} \sum_{m=1}^{M} d_{w}^{m} \ln d_{w}^{m} .
\end{aligned}
$$

Substituting (16) and (17) into (49), respectively, and in view of (1), the minimization of TPTT can be formulated as the following minimization problem under time-based criterion and monetary-based criterion, respectively.

$$
\begin{aligned}
& \min _{f \in \Omega_{f}} F^{t}(f)=\sum_{a \in A} t_{a}\left(v_{a}\right) v_{a}+\sum_{w \in W} \sum_{r \in R_{w}} \sum_{m=1}^{M} c f_{r w} f_{r w}^{m}+\frac{1}{\theta} \sum_{w \in W} \sum_{r \in R_{w}} \sum_{m=1}^{M} f_{r w}^{m} \ln f_{r w}^{m}-\frac{1}{\theta} \sum_{w \in W} \sum_{m=1}^{M} d_{w}^{m} \ln d_{w}^{m} . \\
& \min _{f \in \Omega_{f}} F^{c}(f)=\sum_{a \in A} \sum_{m=1}^{M} \beta_{m} t_{a}\left(v_{a}\right) v_{a}^{m}+\sum_{w \in W} \sum_{r \in R_{w}} \sum_{m=1}^{M} c f_{r w} f_{r w}^{m}+\frac{1}{\theta} \sum_{w \in W} \sum_{r \in R_{w}} \sum_{m=1}^{M} f_{r w}^{m} \ln f_{r w}^{m}-\frac{1}{\theta} \sum_{w \in W} \sum_{m=1}^{M} d_{w}^{m} \ln d_{w}^{m} .
\end{aligned}
$$

Let $\hat{f} \in \Omega_{f}$ be path flow solution of problem (50), the corresponding link flow solution is denoted by $\hat{v} \in \Omega_{v}$. Then, the minimum TPTT is denoted by $F_{\text {CL-SSO }}^{t}=F^{t}(\hat{f})$. Let $\breve{f} \in \Omega_{f}$ be path flow solution of problem (51), the corresponding link flow solution is denoted by $\breve{v} \in \Omega_{\gamma}$. Then, the minimum total perceived travel cost (TPTC) is denoted by $F_{\text {CL-SSO }}^{c}=F^{c}(\breve{f})$. They are given by

$$
\begin{aligned}
F_{\mathrm{CL}-\mathrm{SSO}}^{t} & =\sum_{a \in A} t_{a}\left(\widehat{v}_{a}\right) \widehat{v}_{a}+\sum_{w \in W} \sum_{r \in R_{w}} \sum_{m=1}^{M} c f_{r w} \widehat{f}_{r w}^{m}+\frac{1}{\theta} \sum_{w \in W} \sum_{r \in R_{w}} \sum_{m=1}^{M} \widehat{f}_{r w}^{m} \ln \widehat{f}_{r w}^{m}-\frac{1}{\theta} \sum_{w \in W} \sum_{m=1}^{M} d_{w}^{m} \ln d_{w}^{m} . \\
F_{\mathrm{CL}-\mathrm{SSO}}^{c} & =\sum_{a \in A} \sum_{m=1}^{M} \beta_{m} t_{a}\left(\breve{v}_{a}\right) \breve{v}_{a}^{m}+\sum_{w \in W} \sum_{r \in R_{w}} \sum_{m=1}^{M} c f_{r w} \stackrel{\sim}{f}_{r w}^{m}+\frac{1}{\theta} \sum_{w \in W} \sum_{r \in R_{w}} \sum_{m=1}^{M} \breve{f}_{r w}^{m} \ln \breve{f}_{r w}^{m}-\frac{1}{\theta} \sum_{w \in W} \sum_{m=1}^{M} d_{w}^{m} \ln d_{w}^{m} .
\end{aligned}
$$

Correspondingly, let $F_{\mathrm{CL}-\mathrm{SUE}}^{t}$ and $F_{\mathrm{CL}-\mathrm{SUE}}^{c}$ denote the TPTT and the TPTC at the multiclass CL-SUE, respectively.

Then, we have

$$
\begin{aligned}
& F_{\mathrm{CL}-\mathrm{SUE}}^{t}=\sum_{a \in A} t_{a}\left(\bar{v}_{a}^{t}\right) \bar{v}_{a}^{t}+\sum_{w \in W} \sum_{r \in R_{w}} \sum_{m=1}^{M} c f_{r w} \bar{f}_{r w}^{m, t}+\frac{1}{\theta} \sum_{w \in W} \sum_{r \in R_{w}} \sum_{m=1}^{M} \bar{f}_{r w}^{m, t} \ln \bar{f}_{r w}^{m, t}-\frac{1}{\theta} \sum_{w \in W} \sum_{m=1}^{M} d_{w}^{m} \ln d_{w}^{m}, \\
& F_{\mathrm{CL}-\mathrm{SUE}}^{c}=\sum_{a \in A} \sum_{m=1}^{M} \beta_{m} t_{a}\left(\bar{v}_{a}^{c}\right) \bar{v}_{a}^{m, c}+\sum_{w \in W} \sum_{r \in R_{w}} \sum_{m=1}^{M} c f_{r w} \bar{f}_{r w}^{m, c}+\frac{1}{\theta} \sum_{w \in W} \sum_{r \in R_{w}} \sum_{m=1}^{M} \bar{f}_{r w}^{m, c} \ln \bar{f}_{r w}^{m, c}-\frac{1}{\theta} \sum_{w \in W} \sum_{m=1}^{M} d_{w}^{m} \ln d_{w}^{m} .
\end{aligned}
$$


By definition, we easily have $F_{\mathrm{CL}-\mathrm{SUE}}^{t} \geq F_{\mathrm{CL}-\mathrm{SSO}}^{t}$ and $F_{\mathrm{CL}-S U E}^{c} \geq F_{\mathrm{CL}-S S O}^{c}$. However, it can be found from (52)-(54) that $F_{\mathrm{CL}-S S O}^{t}, F_{\mathrm{CL}-S S O}^{c}, F_{\mathrm{CL}-\mathrm{SUE}}^{t}$, and $F_{\mathrm{CL}-\mathrm{SUE}}^{c}$ may be negative, which implies that the ratio $F_{\mathrm{CL}-\mathrm{SUE}}^{t} / F_{\mathrm{CL}-\mathrm{SSO}}^{t}$ and $F_{\mathrm{CL}-\mathrm{SUE}}^{c} / F_{\mathrm{CL}-\mathrm{SSO}}^{c}$ may be meaningless. Therefore, instead of using the ratio $F_{\mathrm{CL}-\mathrm{SUE}}^{t} / F_{\mathrm{CL}-\mathrm{SSO}}^{t}$ and $F_{\mathrm{CL}-\mathrm{SUE}}^{c} / F_{\mathrm{CL}-\mathrm{SSO}}^{c}$, we use the terms $F_{\mathrm{CL}-S U E}^{t}-F_{\mathrm{CL}-S S O}^{t} \geq 0$ and $F_{\mathrm{CL}-S U E}^{c}-F_{\mathrm{CL}-S S O}^{c} \geq 0$ to quantify the absolute inefficiency of the multiclass CLSUE against the multiclass CL-SSO under time-based criterion and monetary-based criterion, respectively. They are defined as

$$
\begin{aligned}
& \rho_{\mathrm{CL}-\mathrm{SUE} / \mathrm{CL}-\mathrm{SSO}}^{t}=F_{\mathrm{CL}-\mathrm{SUE}}^{t}-F_{\mathrm{CL}-\mathrm{SSO}}^{t}, \\
& \rho_{\mathrm{CL}-\mathrm{SUE} / \mathrm{CL}-\mathrm{SSO}}^{c}=F_{\mathrm{CL}-\mathrm{SUE}}^{c}-F_{\mathrm{CL}-\mathrm{SSO}}^{c} .
\end{aligned}
$$

4.1. Time Units. Setting $\bar{f}=\bar{f}^{t}$ and $f=\widehat{f}$ in VI (18), we can obtain inequality as follows:

$$
\sum_{a \in A} t_{a}\left(\bar{v}_{a}^{t}\right)\left(\bar{v}_{a}-\bar{v}_{a}^{t}\right)+\frac{1}{\theta} \sum_{w \in W} \sum_{r \in R_{w}} \sum_{m=1}^{M}\left(\ln \bar{f}_{r w}^{m, t}+\theta c f_{r w}\right)\left(\hat{f}_{r w}^{m}-\bar{f}_{r w}^{m, t}\right) \geq 0
$$

This leads to

$$
\begin{aligned}
\sum_{a \in A} t_{a}\left(\bar{v}_{a}^{t}\right) \bar{v}_{a}^{t}+\sum_{w \in W} \sum_{r \in R_{w}} \sum_{m=1}^{M} c f_{r w} \bar{f}_{r w}^{m, t}+\frac{1}{\theta} \sum_{w \in W} \sum_{r \in R_{w}} \sum_{m=1}^{M} \bar{f}_{r w}^{m, t} \ln \bar{f}_{r w}^{m, t} \leq & \sum_{a \in A} t_{a}\left(\hat{v}_{a}\right) \widehat{v}_{a}+\sum_{w \in W} \sum_{r \in R_{w}} \sum_{m=1}^{M} c f_{r w} \hat{f}_{r w}^{m} \\
& +\frac{1}{\theta} \sum_{w \in W} \sum_{r \in R_{w}} \sum_{m=1}^{M} \tilde{f}_{r w}^{m} \ln \hat{f}_{r w}^{m}+\sum_{a \in A}\left(t_{a}\left(\bar{v}_{a}^{t}\right)-t_{a}\left(\hat{v}_{a}\right)\right) \hat{v}_{a} \\
& +\frac{1}{\theta} \sum_{w \in W} \sum_{r \in R_{w}} \sum_{m=1}^{M} \tilde{f}_{r w}^{m}\left(\ln \bar{f}_{r w}^{m, t}-\ln \hat{f}_{r w}^{m}\right) .
\end{aligned}
$$

Thus,

$$
F_{\mathrm{CL}-\mathrm{SUE}}^{t}-F_{\mathrm{CL}-\mathrm{SSO}}^{t} \leq \sum_{a \in A}\left(t_{a}\left(\bar{v}_{a}^{t}\right)-t_{a}\left(\widehat{v}_{a}\right)\right) \widehat{v}_{a}+\frac{1}{\theta} \sum_{w \in W} \sum_{r \in R_{w}} \sum_{m=1}^{M} \hat{f}_{r w}^{m}\left(\ln \bar{f}_{r w}^{m, t}-\ln \widehat{f}_{r w}^{m}\right) .
$$

We can get the upper bound of the term $\sum_{a \in A}\left(t_{a}\left(\bar{v}_{a}^{t}\right)-\right.$ $\left.t_{a}\left(\widehat{v}_{a}\right)\right) \hat{v}_{a}$ in (58) from Lemma 3 . Let $v_{a}=\widehat{v}_{a}$, we can obtain inequality below.

$$
\sum_{a \in A}\left(t_{a}\left(\bar{v}_{a}^{t}\right)-t_{a}\left(\widehat{v}_{a}\right)\right) \widehat{v}_{a} \leq \gamma(\varphi) T_{\mathrm{CL}-\mathrm{SUE}}^{t} .
$$

The upper bound on the last term of the right-hand side of (58) is equal to zero from Gibbs' inequality. Therefore, the following inequality holds:

$$
\sum_{r \in R_{w}} \widehat{f}_{r w}^{m}\left(\ln \bar{f}_{r w}^{m, t}-\ln \widetilde{f}_{r w}^{m}\right) \leq 0, w \in W, m \in M
$$

Here, $\sum_{r \in R_{w}} \tilde{f}_{r w}^{m}\left(\ln \bar{f}_{r w}^{m, t}-\ln \tilde{f}_{r w}^{m}\right)=0$ if and only if $\bar{f}_{r w}^{m, t}=\tilde{f}_{r w}^{m}$. Substituting (59) and (60) into (58), the following theorem can be obtained.
Theorem 3. Let $\varphi$ denote a class of differentiable, separable, convex, and monotonically increasing link time functions $t_{a}\left(v_{a}\right)$. Then, the absolute inefficiency of the multiclass $C L-$ SUE against the multiclass CL-SSO under time-based criterion, $\rho_{C L-S U E / C L-S S O}^{t}$, is upper bounded, i.e.,

$$
\rho_{\mathrm{CL}-\mathrm{SUE} / \mathrm{CL}-\mathrm{SSO}}^{t}=F_{\mathrm{CL}-\mathrm{SUE}}^{t}-F_{\mathrm{CL}-\mathrm{SSO}}^{t} \leq \gamma(\varphi) T_{\mathrm{CL}-\mathrm{SUE}}^{t} .
$$

We now begin to discuss the tightness of the bound given in (61) and provide Corollary 2 (the similar process of proof is shown in Guo et al. [37]).

Corollary 2. $F_{C L-S U E}^{t}-F_{C L-S S O}^{t}=\gamma(\varphi) T_{C L-S U E}^{t}$ if and only if $\gamma(\varphi)=0$.

Corollary 2 It states that the upper bound (61) is tight if and only if $\chi(\varphi)=0$ (without traffic congestion). Here, we have $\vec{f}^{t}=f$ and $F_{\mathrm{CL}-\mathrm{SUE}}^{t}-F_{\mathrm{CL}-\mathrm{SSO}}^{t}$. However, traffic 
congestion is a common phenomenon in a realistic transportation network. As a result, we always have $F_{\mathrm{CL}-S U E}^{t}-F_{\mathrm{CL}-S S O}^{t}<\gamma(\varphi) T_{\mathrm{CL}-\mathrm{SUE}}^{t}$, which means that the upper bound (61) is usually not tight in most cases.
4.2. Monetary Units. Setting $\bar{f}=\bar{f}^{c}$ and $f=\breve{f}$ in VI (18), we can obtain the inequality as follows:

$$
\sum_{a \in A} \sum_{m=1}^{M} \beta_{m} t_{a}\left(\bar{v}_{a}^{c}\right)\left(\breve{v}_{a}^{m}-\bar{v}_{a}^{m, c}\right)+\frac{1}{\theta} \sum_{w \in W} \sum_{r \in R_{w}} \sum_{m=1}^{M}\left(\ln \bar{f}_{r w}^{m, c}+\theta c f_{r w}\right)\left(\breve{f}_{r w}^{m}-\bar{f}_{r w}^{m, c}\right) \geq 0
$$

This leads to

$$
\begin{aligned}
\sum_{a \in A} \sum_{m=1}^{M} \beta_{m} t_{a}\left(\bar{v}_{a}^{c}\right) \bar{v}_{a}^{m, c}+\sum_{w \in W} \sum_{r \in R_{w}} \sum_{m=1}^{M} c f_{r w} \bar{f}_{r w}^{m, c}+\frac{1}{\theta} \sum_{w \in W} \sum_{r \in R_{w}} \sum_{m=1}^{M} \widetilde{f}_{r w}^{m, c} \ln \bar{f}_{r w}^{m, c} \leq & \sum_{a \in A} \sum_{m=1}^{M} \beta_{m} t_{a}\left(\breve{v}_{a}\right) \breve{v}_{a}^{m}+\sum_{w \in W} \sum_{r \in R_{w}} \sum_{m=1}^{M} c f_{r w} \breve{f}_{r w}^{m} \\
& +\frac{1}{\theta} \sum_{w \in W} \sum_{r \in R_{w}} \sum_{m=1}^{M} \breve{f}_{r w}^{m} \ln \breve{f}_{r w}^{m} \\
& +\sum_{a \in A} \sum_{m=1}^{M} \beta_{m} \breve{v}_{a}^{m}\left(t_{a}\left(\bar{v}_{a}^{c}\right)-t_{a}\left(\breve{v}_{a}\right)\right) \\
& +\frac{1}{\theta} \sum_{w \in W} \sum_{r \in R_{w}} \sum_{m=1}^{M} \breve{f}_{r w}^{m}\left(\ln \breve{f}_{r w}^{m, c}-\ln \breve{f}_{r w}^{m}\right) .
\end{aligned}
$$

Thus,

$$
F_{\mathrm{CL}-\mathrm{SUE}}^{c}-F_{\mathrm{CL}-\mathrm{SSO}}^{c} \leq \sum_{a \in A} \sum_{m=1}^{M} \beta_{m} \stackrel{\sim}{v}_{a}^{m}\left(t_{a}\left(\bar{v}_{a}^{c}\right)-t_{a}\left(\breve{v}_{a}\right)\right)+\frac{1}{\theta} \sum_{w \in W} \sum_{r \in R_{w}} \sum_{m=1}^{M} \breve{f}_{r w}^{m}\left(\ln \bar{f}_{r w}^{m, c}-\ln \breve{f}_{r w}^{m}\right) .
$$

We can get the upper bound of the term $\sum_{a \in A} \sum_{m=1}^{M} \beta_{m} v_{a}^{m}\left(t_{a}\left(\bar{v}_{a}^{c}\right)-t_{a}\left(\bar{v}_{a}\right)\right)$ in (64) by applying the definitions (39)-(42). Let $v_{a}$ be $v_{a}$ and $z_{a}$ be $\bar{v}_{a}^{c}$, then we have

$$
\sum_{a \in A} \sum_{m=1}^{M} \beta_{m} \breve{v}_{a}^{m}\left(t_{a}\left(\bar{v}_{a}^{c}\right)-t_{a}\left(\breve{v}_{a}\right)\right)=\sum_{a \in A_{1}} \sum_{m=1}^{M} \beta_{m} \breve{v}_{a}^{m}\left(t_{a}\left(\bar{v}_{a}^{c}\right)-t_{a}\left(\breve{v}_{a}\right)\right)+\sum_{a \notin A_{1}} \sum_{m=1}^{M} \beta_{m} \stackrel{-v}{v}_{a}^{m}\left(t_{a}\left(\bar{v}_{a}^{c}\right)-t_{a}\left(\breve{v}_{a}\right)\right) \leq \bar{\gamma}(\varphi, \beta) T_{\mathrm{CL}-\mathrm{SUE}}^{c}+\tilde{\gamma}(\varphi, \beta) T_{\mathrm{CL}-\mathrm{SSO}}^{c}
$$

where $T_{\mathrm{CL}-\mathrm{SSO}}^{c}=\sum_{a \in A} \sum_{m=1}^{M} \beta_{m} t_{a}\left(\breve{v}_{a}\right) \breve{v}_{a}^{m}$ is the TSTC at the multiclass CL-SSO.

The upper bound on the last term of the right-hand side of (64) is equal to zero from Gibbs' inequality. Therefore, the following inequality holds:

$$
\sum_{r \in R_{w}} \breve{f}_{r w}^{m}\left(\ln \bar{f}_{r w}^{m, c}-\ln \breve{f}_{r w}^{m}\right) \leq 0, w \in W, m \in M .
$$

Here, $\sum_{r \in R_{w}} \breve{f}_{r w}^{m}\left(\ln \bar{f}_{r w}^{m, c}-\ln \breve{f}_{r w}^{m}\right)=0$ if and only if $\bar{f}_{r w}^{m, c}=\breve{f}_{r w}^{m}$. Substituting (65) and (66) into (64), we can obtain the theorem as follows.
Theorem 4. Let $\varphi$ denote a class of differentiable, separable, convex, and monotonically increasing link time functions $t_{a}\left(v_{a}\right)$. Then, the absolute inefficiency of the multiclass CLSUE against the multiclass CL-SSO under monetary-based criterion, $\rho_{C L-S U E / C L-S S O}^{c}$, is upper bounded, i.e.,

$$
\begin{aligned}
\rho_{\mathrm{CL}-\mathrm{SUE} / \mathrm{CL}-\mathrm{SSO}}^{c} & =F_{\mathrm{CL}-\mathrm{SUE}}^{c}-F_{\mathrm{CL}-\mathrm{SSO}}^{c} \\
& \leq \bar{\gamma}(\varphi, \beta) T_{\mathrm{CL}-\mathrm{SUE}}^{c}+\tilde{\gamma}(\varphi, \beta) T_{\mathrm{CL}-\mathrm{SSO}}^{c} .
\end{aligned}
$$

Next, we will discuss the tightness of the bound given in (67) and provide Corollary 3 as follows. 
Corollary 3. $F_{C L-S U E}^{c}-F_{C L-S S O}^{c}=\bar{\gamma}(\varphi, \beta) T_{C L-S U E}^{c}+\tilde{\gamma}(\varphi$, $\beta) T_{C L-S S O}^{c}$ if and only if $\bar{\gamma}(\varphi, \beta)=\widetilde{\gamma}(\varphi, \beta)=0$.

Proof of Corollary 3. Firstly, if $\bar{\gamma}(\varphi, \beta)=\widetilde{\gamma}(\varphi, \beta)=0$, we can obtain $F_{\mathrm{CL}-\mathrm{SUE}}^{c}-F_{\mathrm{CL}-\mathrm{SSO}}^{c} \leq 0$ from Theorem 4 . According to the definition of the multiclass CL-SSO, we have $F_{\text {CL-SUE }}^{c}$ $\geq F_{\mathrm{CL}-\mathrm{SSO}}^{c}$. Hence, we can obtain $F_{\mathrm{CL}-\mathrm{SUE}}^{c}=F_{\mathrm{CL}-\mathrm{SSO}}^{c}$, which means that $F_{\mathrm{CL}-\mathrm{SUE}}^{c}-F_{\mathrm{CL}-\mathrm{SSO}}^{c}=0$. Since $\bar{\gamma}(\varphi, \beta)=\tilde{\gamma}(\varphi, \beta)=$ 0 , we have $\bar{\gamma}(\varphi, \beta) T_{\mathrm{CL}-S U E}^{c}+\widetilde{\gamma}(\varphi, \beta) T_{\mathrm{CL}-\mathrm{SSO}}^{c}=0$. Therefore, $F_{\mathrm{CL}-\mathrm{SUE}}^{c}-F_{\mathrm{CL}-\mathrm{SSO}}^{c}=\bar{\gamma}(\varphi, \beta) T_{\mathrm{CL}-\mathrm{SUE}}^{c}+\widetilde{\gamma}(\varphi, \beta) T_{\mathrm{CL}-\mathrm{SSO}}^{c}$ if $\bar{\gamma}$ $(\varphi, \beta)=\tilde{\gamma}(\varphi, \beta)=0$. Secondly, we assume that $F_{\mathrm{CL}-\mathrm{SUE}}^{c}-$ $F_{\mathrm{CL}-S S O}^{c}=\bar{\gamma}(\varphi, \beta) T_{\mathrm{CL}-S \mathrm{SUE}}^{c}+\tilde{\gamma}(\varphi, \beta) T_{\mathrm{CL}-\mathrm{SSO}}^{c}$ holds. From the specific derivation process of (67), $F_{\mathrm{CL}-\mathrm{SUE}}^{c}-F_{\mathrm{CL}-\mathrm{SSO}}^{c}$ $=\bar{\gamma}(\varphi, \beta) T_{\mathrm{CL}-\mathrm{SUE}}^{c}+\tilde{\gamma}(\varphi, \beta) T_{\mathrm{CL}-\mathrm{SSO}}^{c}$ implies that inequality (66) takes equality; i.e., $\bar{f}^{c}=\breve{f}$. Since $\bar{f}^{c}=\breve{f}$, we can have $\bar{v}^{c}=\breve{v}, F_{\mathrm{CL}-\mathrm{SUE}}^{c}=F_{\mathrm{CL}-\mathrm{SSO}}^{c}$, and $T_{\mathrm{CL}-\mathrm{SUE}}^{c}=T_{\mathrm{CL}-\mathrm{SSO}}^{c}$. With definitions (39)-(42), if $\bar{v}^{c}=\breve{v}$ holds, we can obtain $\bar{\gamma}(\varphi, \beta)=\widetilde{\gamma}(\varphi, \beta)=0$. The proof is completed.

Corollary 3 shows that the upper bound (67) is tight if and only if $\bar{\gamma}(\varphi, \beta)=\widetilde{\gamma}(\varphi, \beta)=0$. The expression $\bar{\gamma}(\varphi, \beta)=$ $\tilde{\gamma}(\varphi, \beta)=0$ means that the multiclass CL-SUE state is equivalent to the multiclass CL-SSO state. Here, we have $\bar{f}^{c}=f, \bar{v}^{c}=\breve{v}, F_{\mathrm{CL}-\mathrm{SUE}}^{c}=F_{\mathrm{CL}-\mathrm{SSO}}^{c}$, and $T_{\mathrm{CL}-\mathrm{SUE}}^{c}=T_{\mathrm{CL}-\mathrm{SSO}}^{c}$, which are usually not satisfied in a realistic transportation network. As a result, we always have $F_{\mathrm{CL}-\mathrm{SUE}}^{c}-F_{\mathrm{CL}-\mathrm{SSO}}^{c}$ $<\bar{\gamma}(\varphi, \beta) T_{\mathrm{CL}-S U E}^{c}+\tilde{\gamma}(\varphi, \beta) T_{\mathrm{CL}-S S O}^{c}$, which means that the upper bound (67) is usually not tight in most cases.

\section{Effects of Parameters on the Inefficiency Bounds}

This section discusses the effects of various parameters on the upper bounds (33), (48), (61), and (67), namely, $\gamma(\varphi)$, $\bar{\gamma}(\varphi, \beta), \widetilde{\gamma}(\varphi, \beta), \bar{k}, \theta, \bar{c}$, and $\widetilde{c}$.

$\gamma(\varphi) \in[0,1]$ is a dimensionless coefficient defined only by the class of link time functions. Both the upper bounds (33) and (61) are monotonically increasing functions of $\gamma(\varphi)$. Consider a widely used class of link travel time functions, $t_{a}\left(v_{a}\right)=t_{a}^{0}+\alpha_{a}\left(v_{a}\right)^{p}, a \in A$, where $t_{a}^{0} \geq 0$ denotes free-flow travel time on link $a \in A, \alpha_{a} \geq 0$ is a constant, and $p \geq 0$ reflects the degree of traffic congestion. Roughgarden [27] provided a specific expression of $\gamma(\varphi)$ as follows:

$$
\gamma(\varphi)=\left(\frac{p}{p+1}\right)\left(\frac{1}{p+1}\right)^{1 / p} .
$$

Equation (68) shows that $\gamma(\varphi) \longrightarrow 0$ when $p \longrightarrow 0$ (without traffic congestion) and $\gamma(\varphi) \longrightarrow 0$ when $p \longrightarrow$ $+\infty$ (with severe congestion). When $p=1$ and $4, \gamma(\varphi)=$ 0.25 and 0.535 , respectively. So, the upper bounds (33) and (61) are both monotonically increasing functions of $p$. When the value of $p$ can be reduced, we will have more space to improve the congestion in the traffic network by driving the multiclass CL-SUE state to the multiclass SO or the multiclass CL-SSO state.

Both $\bar{\gamma}(\varphi, \beta)$ and $\tilde{\gamma}(\varphi, \beta)$ are dimensionless coefficients depending on the class of link time functions and VOT $\beta$.
Both the upper bounds (48) and (67) are monotonically increasing functions of $\bar{\gamma}(\varphi, \beta)$ and $\tilde{\gamma}(\varphi, \beta)$.

Recall that $k_{w}^{\mathrm{CL}-\mathrm{SUE}}$ solves the equation $k \exp (k+1)=$ $\sum_{r=1, r \neq j}^{\left|R_{w}\right|} \exp \left(\theta\left(c f_{j w}-c f_{r w}\right)\right) \quad$ with $j=\arg \max r\left(c f_{r w}, r\right.$ $\left.\in R_{w}\right), w \in W$, and $\bar{k}=\sum_{w \in W} \sum_{m=1}^{M}\left(d_{w}^{m} / D\right) k_{w}^{\mathrm{CL}-S U E}$. So, $\bar{k}$ is a dimensionless coefficient, which increases with the relative values of the commonality factors of paths and the number of feasible paths. Hence, $\bar{k}$ can reflect the degree of network complexity. Let $j^{\prime}=\arg \min r\left(c f_{r w}, r \in R_{w}\right), w \in W$, then we have $k_{w}^{\mathrm{CL}-S U E} \exp \left(k_{w}^{\mathrm{CL}-S U E}+1\right) \leq\left(\left|R_{w}\right|-1\right) \exp \left(\theta\left(c f_{j w}\right.\right.$ $\left.\left.-c f_{j^{\prime} w}\right)\right)$. If each OD pair has only one available path in a transportation network, i.e., $\left|R_{w}\right|=1$, we can obtain $k_{w}^{\mathrm{CL}-\mathrm{SUE}}=0$ and thus $\bar{k}=0$. When $\left|R_{w}\right|=1$, we have $\rho_{\text {CL-SUE }}^{t} \leq(1-\gamma(\varphi))^{-1}$ and $\rho_{\text {CL-SUE }}^{c} \leq(1+\widetilde{\gamma}(\varphi, \beta)) /(1-\bar{\gamma}$ $(\varphi, \beta))$. When $\left|R_{w}\right|>1$, the value of $k_{w}^{\mathrm{CL}-\mathrm{SUE}}$ (and hence $\bar{k}$ ) is greatly limited only if values of $\theta$ and $\mathrm{cf}_{r w}$ are given (the similar discussion is shown in Guo et al. [37]). Even though the effect is small, both the upper bounds (33) and (48) are increasing with the degree of network complexity.

Parameter $\theta$ is the perception error of travel time, which can reflect the degree of network familiarity. Both the upper bounds (33) and (48) decrease with $\theta$. When $\theta \longrightarrow+\infty$, we have $\rho_{\text {CL-SUE }}^{t} \leq(1-\gamma(\varphi))^{-1}$ and $\rho_{\text {CL-SUE }}^{c} \leq(1+\widetilde{\gamma}(\varphi, \beta)) /(1-$ $\bar{\gamma}(\varphi, \beta))$, which are the results of the standard multiclass UE problem under time-based criterion and monetary-based criterion, respectively.

Theorems 1 and 2 show that the upper bound (33) decreases with $\bar{c}$ and the upper bound (48) decreases with $\tilde{c}$, respectively. Since $\bar{c}=T_{\mathrm{SO}}^{t} / D$ and $\widetilde{c}=T_{\mathrm{SO}}^{c} / D$, both the upper bounds (33) and (48) will increase when the total demand, $D$, goes up.

Finally, the results of the above discussions are summarized in Table 2.

\section{Summary of the Main Research Findings}

In Sections 3 and 4, we have derived four inefficiency bounds of the multiclass CL-SUE, and given four theorems. In this section, we provide a table to summarize the main research findings of four theorems in this article. These findings are shown in Table 3.

\section{Numerical Example}

The Nguyen and Dupuis network [50] shown in Figure 2 is used to further illustrate the above conclusions. The network consists of 19 links, 13 nodes, 25 paths, and 4 OD pairs. All users are classified on the basis of different VOTs into three main classes. Let $\beta_{1}=1.0$ (¥/min) (denoted by “1”) be VOT for users of class $1, \beta_{2}=2.0$ (¥/min) (denoted by "2") be VOT for users of class 2 , and $\beta_{3}=3.0(¥ / \mathrm{min})$ (denoted by " 3 ") be VOT for users of class 3. As shown in Figure 2, the network has 4 OD pairs: $1 \longrightarrow 2$ (denoted by " $w_{1}$ "), $1 \longrightarrow 3$ (denoted by " $w_{2}$ "), $4 \longrightarrow 2$ (denoted by " $w_{3}$ "), and $4 \longrightarrow 3$ (denoted by " $w_{4}$ "). Suppose that the traffic demand is $d_{w_{1}}^{1}=120, \quad d_{w_{1}}^{2}=200, \quad d_{w_{1}}^{3}=80, \quad d_{w_{2}}^{1}=240, \quad d_{w_{2}}^{2}=400$, 
TABLE 2: Effects of various parameters on the upper bounds.

\begin{tabular}{lcccccc}
\hline & Congestion degree & $\bar{\gamma}(\varphi, \beta)$ & $\tilde{\gamma}(\varphi, \beta)$ & Network complexity & Network familiarity & Total demand \\
\hline Bound (33) & + & No & No & + & - & + \\
Bound (48) & No & + & + & + & - & No \\
Bound (61) & + & No & No & No & No & No \\
Bound (67) & No & + & + & No & No \\
\hline
\end{tabular}

TABLE 3: The main research findings of four theorems.

\begin{tabular}{lll}
\hline Theorem & The upper bounds & Main research findings
\end{tabular}

(1) The upper bound (33) depends on four parameters, namely, $\gamma(\varphi), \bar{k}, \theta$, and $\bar{c}(2)$ Corollary 1 : in a transportation network, the efficiency loss upper bound against the multiclass SO by the

Theorem $1 \quad \rho_{\mathrm{CL}-\mathrm{SUE}}^{t}=T_{\mathrm{CL}-\mathrm{SUE}}^{t} / T_{\mathrm{SO}}^{t} \leq(1 /(1-\gamma(\varphi)))(1+\bar{k} /(\theta \bar{c}))$ multiclass CL-SUE under time-based criterion is not less than that by the multiclass SUE. Corollary 1 shows that the multiclass CL-SUE model provides a more realistic upper bound than the multiclass SUE model by considering the path overlapping problem.

(1) The upper bound (48) depends on five parameters, namely, $\bar{\gamma}(\varphi, \beta), \tilde{\gamma}(\varphi, \beta), \bar{k}, \theta$, and $\widetilde{c}$. (2) Similar to the proof of Corollary

Theorem $2 \rho_{\mathrm{CL}-\mathrm{SUE}}^{c}=T_{\mathrm{CL}-\mathrm{SUE}}^{c} / T_{\text {SO }}^{c} \leq(1+\widetilde{\gamma}(\varphi, \beta)+\bar{k} /(\theta \widetilde{c})) /(1-\bar{\gamma}(\varphi, \beta)) \quad 1$, we can find that the efficiency loss upper bound against the multiclass SO by the multiclass CL-SUE under monetary-based criterion is not less than that by the multiclass SUE.

(1) The upper bound (61) depends on two parameters, namely,

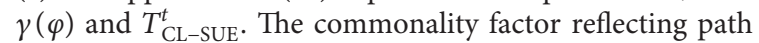
overlapping has no impact on the inefficiency upper bound

Theorem $3 \quad \rho_{\mathrm{CL}-\mathrm{SUE} / \mathrm{CL}-\mathrm{SSO}}^{t}=F_{\mathrm{CL}-\mathrm{SUE}}^{t}-F_{\mathrm{CL}-\mathrm{SSO}}^{t} \leq \gamma(\varphi) T_{\mathrm{CL}-\mathrm{SUE}}^{t}$ (61).(2) Corollary $2\left(F_{\mathrm{CL}-\mathrm{SUE}}^{t}-F_{\mathrm{CL}-\mathrm{SSO}}^{t}=\gamma(\varphi) T_{\mathrm{CL}-\mathrm{SUE}}^{t}\right.$ if and only if $\gamma(\varphi)=0)$. It states that the upper bound (61) is tight if and only if $\gamma(\varphi)=0$ (without traffic congestion), which means that the upper bound (61) is usually not tight in most cases.

(1) The upper bound (67) depends on four parameters, namely, $\bar{\gamma}(\varphi, \beta), \tilde{\gamma}(\varphi, \beta), T_{\mathrm{CL}-\mathrm{SUE}}^{c}$, and $T_{\mathrm{CL}-\mathrm{SSO}}^{c}$. The commonality factor reflecting path overlapping has no impact on the inefficiency upper bound (67).(2) Corollary 3

$\rho_{\mathrm{CL}-\mathrm{SUE} / \mathrm{CL}-\mathrm{SSO}}^{c}=$
Theorem $4 \quad F_{\mathrm{CL}-\mathrm{SUE}}^{c}-F_{\mathrm{CL}-\mathrm{SSO}}^{c} \leq \bar{\gamma}(\varphi, \beta) T_{\mathrm{CL}-\mathrm{SUE}}^{c}$
$+\widetilde{\gamma}(\varphi, \beta) T_{\mathrm{CL}-\mathrm{SSO}}^{c}$

$\left(F_{\mathrm{CL}-\mathrm{SUE}}^{c}-F_{\mathrm{CL}-\mathrm{SSO}}^{c}=\bar{\gamma}(\varphi, \beta) T_{\mathrm{CL}-\mathrm{SUE}}^{c}+\widetilde{\gamma}(\varphi, \beta) T_{\mathrm{CL}-\mathrm{SSO}}^{c}\right.$ if and only if $\bar{\gamma}(\varphi, \beta)=\widetilde{\gamma}(\varphi, \beta)=0)$ : It shows that the upper bound (67) is tight if and only if $\bar{\gamma}(\varphi, \beta)=\widetilde{\gamma}(\varphi, \beta)=0$, which means that the upper bound (67) is usually not tight in most cases.

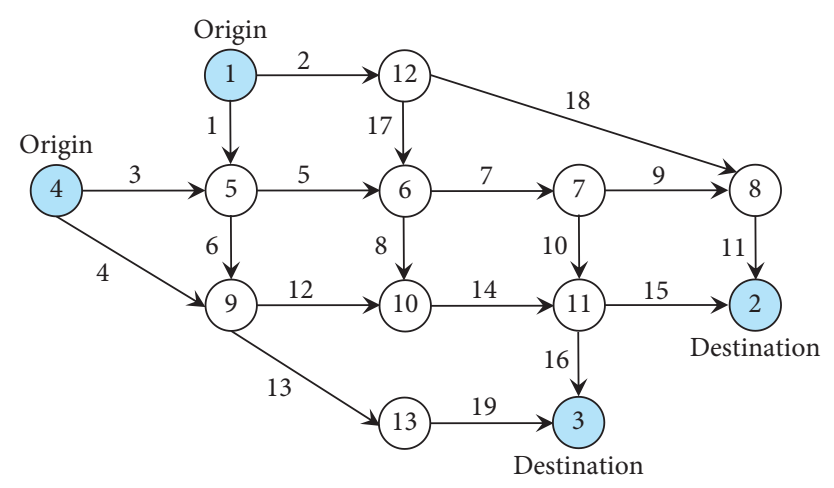

Figure 2: Nguyen and Dupuis network. 
TABle 4: Parameters of links.

\begin{tabular}{lccccc}
\hline Link no. & Free-flow travel time & Capacity & Link no. & Free-flow travel time & Capacity \\
\hline 1 & 7 & 900 & 11 & 10 & 10 \\
2 & 8 & 700 & 12 & 9 & 700 \\
3 & 9 & 700 & 13 & 8 & 600 \\
4 & 14 & 900 & 14 & 15 & 700 \\
5 & 5 & 800 & 16 & 7 & 700 \\
6 & 9 & 600 & 17 & 15 & 700 \\
7 & 5 & 500 & 18 & 11 & 700 \\
8 & 13 & 500 & 19 & & 700 \\
9 & 5 & 300 & & \\
\hline
\end{tabular}

$d_{w_{2}}^{3}=160, \quad d_{w_{3}}^{1}=180, \quad d_{w_{3}}^{2}=300, \quad d_{w_{3}}^{3}=120, \quad d_{w_{4}}^{1}=60$, $d_{w_{4}}^{2}=100$, and $d_{w_{4}}^{3}=40$. Assume that parameters $\theta=0.5$, $\beta_{0}=1$, and $\gamma_{0}=1$.

The link time functions use the following BPR (Bureau of Public Road) form, with free-flow travel time $t_{a}^{0}$ and link capacity $C_{a}$ provided in Table 4 :

$$
t_{a}\left(v_{a}\right)=t_{a}^{0}\left[1+0.15 \times\left(\frac{v_{a}}{C_{a}}\right)^{4}\right]
$$

The relationship between paths and nodes in the network is shown in Table 5. The commonality factor $\mathrm{cf}_{r w}$ can be calculated by (10). In this paper, the free-flow travel time is used instead of length to calculate $\mathrm{cf}_{r w}$. The results are also shown in Table 5 .

The link flow, path flow, and path cost solutions of the multiclass CL-SUE model are shown in Tables 6 and 7 by solving VI (18), respectively. The link flow solutions of the multiclass SO and CL-SSO models are shown in Table 8 by solving the minimization problems (19), (35), (50), and (51).

From Tables 6 and 7, we can obtain $T_{\text {CL-SUE }}^{t}=\sum_{w \in W}$ $\sum_{r \in R_{w}} \sum_{m=1}^{M} c_{r w}^{m}\left(\bar{f}^{t}\right) \bar{f}_{r w}^{m, t}=86316.9550, T_{\mathrm{CL}-\mathrm{SUE}}^{c}=\sum_{w \in W} \sum_{r \in R_{w}}$ $\sum_{m=1}^{M} c_{r w}^{m}\left(\bar{f}^{c}\right) \bar{f}_{r w}^{m, c}=171413.1091, F_{\mathrm{CL}-\mathrm{SUE}}^{t}=74528.8734$, and $F_{\text {CL-SUE }}^{c}=144177.3510$. From Table 8, we can obtain $T_{\text {SO }}^{t}=$ $77678.1637, \quad T_{\mathrm{SO}}^{c}=146623.4458, \quad T_{\mathrm{CL}-\mathrm{SUE}}^{c}=145869.2905$, $F_{\mathrm{CL}-\mathrm{SSO}}^{t}=74296.2072$, and $F_{\mathrm{CL}-\mathrm{SSO}}^{c}=143566.4683$. Thus, we have $\rho_{\mathrm{CL}-\mathrm{SUE}}^{t}=T_{\mathrm{CL}-\mathrm{SUE}}^{t} / T_{\mathrm{SO}}^{t}=1.1112, \quad \rho_{\mathrm{CL}-\mathrm{SUE}}^{c}=T_{\mathrm{CL}-\mathrm{SUE}}^{c} /$ $T_{\mathrm{SO}}^{c}=1.1691, \rho_{\mathrm{CL}-\mathrm{SUE} / \mathrm{CL}-\mathrm{SSO}}^{t}=F_{\mathrm{CL}-\mathrm{SUE}}^{t}-F_{\mathrm{CL}-\mathrm{SSO}}^{t}=232.6662$, and $\rho_{\mathrm{CL}-\mathrm{SUE} / \mathrm{CL}-\mathrm{SSO}}^{c}=F_{\mathrm{CL}-\mathrm{SUE}}^{c}-F_{\mathrm{CL}-\mathrm{SSO}}^{c}=610.8827$.

For the link time function with $p=4$ in this example, it is easy to obtain that $\gamma(\varphi)=0.535$. Since $k_{w}^{\mathrm{CL}-\mathrm{SUE}}$ is the solution of $k \exp (k+1)=\sum_{r=1, r \neq j}^{\left|R_{w}\right|} \exp \left(\theta\left(c f_{j w}-c f_{r w}\right)\right)$ with $j=\arg \max r\left(c f_{r w}, r \in R_{w}\right), w \in W$, then we can obtain $k_{w_{1}}^{\mathrm{CL}-\mathrm{SUE}}=1.0767, k_{w_{2}}^{\mathrm{CL}-\mathrm{SUE}}=0.9412, k_{w_{3}}^{\mathrm{CL}-\mathrm{SUE}}=0.8441$, and $k_{w_{4}}^{\mathrm{CL}-\mathrm{SUE}}=0.9469$. Hence, we can have $\bar{k}=0.9397$ by $\bar{k}=$ $\sum_{w \in W} \sum_{m=1}^{M}\left(d_{w}^{m} / D\right) k_{w}^{\mathrm{CL}-\mathrm{SUE}}$ and $D=\sum_{w \in W} \sum_{m=1}^{M} d_{w}^{m}$. We can also obtain $\bar{c}=38.8391$ and $\tilde{c}=73.3117$ by $\bar{c}=T_{\mathrm{SO}}^{t} / D$ and $\tilde{c}=T_{\mathrm{SO}}^{c} / D$, respectively.

From Table 7, we can have $\bar{v}_{1}^{1, c}=222.2, \bar{v}_{1}^{2, c}=392.4, \bar{v}_{1}^{3, c}$ $=159.5$, and $\bar{v}_{1}^{c}=774.1$. Based on definition $\gamma_{a}\left(z_{a}, t_{a}, \beta\right)=$ [1/ $\left.\left(\left(\sum_{m=1}^{M} \beta_{m} z_{a}^{m}\right) t_{a}\left(z_{a}\right)\right)\right] \max _{v_{a}^{m} \geq 0}\left\{\left(\sum_{m=1}^{M} \beta_{m} v_{a}^{m}\right)\left(t_{a}\left(z_{a}\right)-t_{a}\right.\right.$
TAble 5: Parameters between four OD pairs.

\begin{tabular}{|c|c|c|c|}
\hline OD pair & Path no. & Node sequence & The value of $\mathrm{cf}_{r w}$ \\
\hline \multirow{8}{*}{$1-2$} & 1 & $1-12-8-2$ & 0.8202 \\
\hline & 2 & $1-5-6-7-8-2$ & 1.1226 \\
\hline & 3 & $1-5-6-7-11-2$ & 1.2437 \\
\hline & 4 & $1-5-6-10-11-2$ & 1.2105 \\
\hline & 5 & $1-5-9-10-11-2$ & 1.0206 \\
\hline & 6 & $1-12-6-7-8-2$ & 1.1621 \\
\hline & 7 & $1-12-6-7-11-2$ & 1.2744 \\
\hline & 8 & $1-12-6-10-11-2$ & 1.2439 \\
\hline \multirow{6}{*}{$1-3$} & 9 & $1-5-9-13-3$ & 0.5843 \\
\hline & 10 & $1-5-6-7-11-3$ & 1.0851 \\
\hline & 11 & $1-5-6-10-11-3$ & 1.1507 \\
\hline & 12 & $1-5-9-10-11-3$ & 1.0777 \\
\hline & 13 & $1-12-6-7-11-3$ & 0.9550 \\
\hline & 14 & $1-12-6-10-11-3$ & 1.0409 \\
\hline \multirow{5}{*}{$4-2$} & 15 & $4-9-10-11-2$ & 0.8153 \\
\hline & 16 & $4-5-6-7-8-2$ & 0.7551 \\
\hline & 17 & $4-5-6-7-11-2$ & 1.0217 \\
\hline & 18 & $4-5-6-10-11-2$ & 1.0704 \\
\hline & 19 & $4-5-9-10-11-2$ & 1.0592 \\
\hline \multirow{6}{*}{$4-3$} & 20 & $4-9-13-3$ & 0.6606 \\
\hline & 21 & $4-9-10-11-3$ & 0.9540 \\
\hline & 22 & $4-5-9-13-3$ & 0.9012 \\
\hline & 23 & $4-5-6-7-11-3$ & 0.8922 \\
\hline & 24 & $4-5-6-10-11-3$ & 1.0088 \\
\hline & 25 & $4-5-9-10-11-3$ & 1.1190 \\
\hline
\end{tabular}

$\left.\left.\left(v_{a}\right)\right)\right\}$ and $t_{1}\left(v_{1}\right)=7\left[1+0.15 \times\left(v_{1} / 900\right)^{4}\right]$, we can obtain $\gamma_{1}\left(z_{1}, t_{1}, \beta\right)=\left(\max _{v_{m}^{m} \geq 0}\left\{\left(1 \cdot v_{1}^{1}+2 \cdot v_{1}^{2}+3 \cdot v_{1}^{3}\right)[7.5748-7\right.\right.$ $\left.\left.\left.\left(1+0.15\left(v_{1} / 900\right)^{4}\right)\right]\right\}\right) /((1 \cdot 222.17+2 \cdot 392.44+3 \cdot 159.53)$. 7.5748). When $v_{1}^{1}=0, \quad v_{1}^{2}=0, \quad v_{1}^{3}=517.7044$, and $v_{1}=517.7044$, the above optimization problem reaches at the maximum $\gamma_{1}\left(z_{1}, t_{1}, \beta\right)=0.0635$. By the same way, we obtain $\gamma_{2}\left(z_{2}, t_{2}, \beta\right)=0.0173, \gamma_{3}\left(z_{3}, t_{3}, \beta\right)=0.0212, \gamma_{4}\left(z_{4}, t_{4}\right.$, $\beta)=0.0029, \gamma_{5}\left(z_{5}, t_{5}, \beta\right)=0.0253, \gamma_{6}\left(z_{6}, t_{6}, \beta\right)=0.1694$, $\gamma_{7}\left(z_{7}, t_{7}, \beta\right)=0.0179, \gamma_{8}\left(z_{8}, t_{8}, \beta\right)=0.000012, \gamma_{9}\left(z_{9}, t_{9}, \beta\right)=$ $0.2739, \gamma_{10}\left(z_{10}, t_{10}, \beta\right)=0.0027, \gamma_{11}\left(z_{11}, t_{11}, \beta\right)=0.1540$, $\gamma_{12}\left(z_{12}, t_{12}, \beta\right)=0.0007, \gamma_{13}\left(z_{13}, t_{13}, \beta\right)=0.3058, \gamma_{14}\left(z_{14}, t_{14}\right.$, $\beta)=0.0015, \gamma_{15}\left(z_{15}, t_{15}, \beta\right)=0.0014, \gamma_{16}\left(z_{16}, t_{16}, \beta\right)=0.0003$, $\gamma_{17}\left(z_{17}, t_{17}, \beta\right)=0.0002, \quad \gamma_{18}\left(z_{18}, t_{18}, \beta\right)=0.0099, \quad$ and $\gamma_{19}\left(z_{19}, t_{19}, \beta\right)=0.2002$. Then, we have $\bar{\gamma}(\varphi, \beta)=0.3058$ and $\widetilde{\gamma}(\varphi, \beta)=0$. Thus, bound (33) becomes $\rho_{\mathrm{CL}-\mathrm{SUE}}^{t}=1.1112 \leq$ $(1 / 1-\gamma(\varphi))(1+\bar{k} / \theta \bar{c})=2.2546$ according to Theorem 1 . 
TABLE 6: Path results of the multiclass CL-SUE model.

\begin{tabular}{|c|c|c|c|c|c|c|c|c|c|c|c|c|}
\hline \multirow{4}{*}{ Path no. } & \multicolumn{12}{|c|}{ The multiclass CL-SUE } \\
\hline & \multicolumn{6}{|c|}{ Path flow } & \multicolumn{6}{|c|}{ Path cost } \\
\hline & \multicolumn{3}{|c|}{ Time-based } & \multicolumn{3}{|c|}{ Monetary-based } & \multicolumn{3}{|c|}{ Time-based } & \multicolumn{3}{|c|}{ Monetary-based } \\
\hline & $m=1$ & $m=2$ & $m=3$ & $m=1$ & $m=2$ & $m=3$ & $m=1$ & $m=2$ & $m=3$ & $m=1$ & $m=2$ & $m=3$ \\
\hline 1 & 85.0 & 141.6 & 56.6 & 96.8 & 197.1 & 79.9 & 33.5 & 33.5 & 33.5 & 33.5 & 67.0 & 100.5 \\
\hline 2 & 21.1 & 35.2 & 14.1 & 10.3 & 1.9 & 0.1 & 32.8 & 32.8 & 32.8 & 32.8 & 65.6 & 98.4 \\
\hline 3 & 1.5 & 2.6 & 1.0 & 2.5 & 0.1 & 0.0 & 36.4 & 36.4 & 36.4 & 36.4 & 72.8 & 109.2 \\
\hline 4 & 0.8 & 1.4 & 0.6 & 0.8 & 0.0 & 0.0 & 44.4 & 44.4 & 44.4 & 44.2 & 88.3 & 132.5 \\
\hline 5 & 0.6 & 0.9 & 0.4 & 0.7 & 0.0 & 0.0 & 51.9 & 51.9 & 51.9 & 50.8 & 101.5 & 152.3 \\
\hline 6 & 9.9 & 16.5 & 6.6 & 6.7 & 0.8 & 0.0 & 35.3 & 35.3 & 35.3 & 35.3 & 70.6 & 105.9 \\
\hline 7 & 0.7 & 1.2 & 0.5 & 1.6 & 0.0 & 0.0 & 38.9 & 38.9 & 38.9 & 38.9 & 77.8 & 116.7 \\
\hline 8 & 0.4 & 0.6 & 0.3 & 0.5 & 0.0 & 0.0 & 46.9 & 46.9 & 46.9 & 46.6 & 93.3 & 139.9 \\
\hline 9 & 160.5 & 267.5 & 107.0 & 141.3 & 362.5 & 157.1 & 56.2 & 56.2 & 56.2 & 57.6 & 115.3 & 172.9 \\
\hline 10 & 30.1 & 50.2 & 20.1 & 40.5 & 23.2 & 2.2 & 34.6 & 34.6 & 34.6 & 34.6 & 69.3 & 103.9 \\
\hline 11 & 16.8 & 28.1 & 11.2 & 13.9 & 2.6 & 0.1 & 42.6 & 42.6 & 42.6 & 42.4 & 84.8 & 127.2 \\
\hline 12 & 12.2 & 20.4 & 8.2 & 12.0 & 2.1 & 0.1 & 50.1 & 50.1 & 50.1 & 49.0 & 98.0 & 147.0 \\
\hline 13 & 13.0 & 21.6 & 8.6 & 23.9 & 8.6 & 0.5 & 37.1 & 37.1 & 37.1 & 37.1 & 74.3 & 111.4 \\
\hline 14 & 7.3 & 12.2 & 4.9 & 8.3 & 1.0 & 0.0 & 45.1 & 45.1 & 45.1 & 44.9 & 89.8 & 134.6 \\
\hline 15 & 34.2 & 56.9 & 22.8 & 55.2 & 80.0 & 23.0 & 44.7 & 44.7 & 44.7 & 44.5 & 89.1 & 133.6 \\
\hline 16 & 126.3 & 210.5 & 84.2 & 87.1 & 205.0 & 95.6 & 34.1 & 34.1 & 34.1 & 34.2 & 68.3 & 102.5 \\
\hline 17 & 9.9 & 16.5 & 6.6 & 23.0 & 12.5 & 1.3 & 37.8 & 37.8 & 37.8 & 37.8 & 75.5 & 113.3 \\
\hline 18 & 5.5 & 9.2 & 3.7 & 7.8 & 1.4 & 0.1 & 45.7 & 45.7 & 45.7 & 45.5 & 91.0 & 136.5 \\
\hline 19 & 4.1 & 6.9 & 2.7 & 7.0 & 1.1 & 0.0 & 53.3 & 53.3 & 53.3 & 52.1 & 104.2 & 156.4 \\
\hline 20 & 49.0 & 81.7 & 32.7 & 48.0 & 98.0 & 39.9 & 48.9 & 48.9 & 48.9 & 51.4 & 102.8 & 154.2 \\
\hline 21 & 3.4 & 5.6 & 2.3 & 3.7 & 0.5 & 0.0 & 42.9 & 42.9 & 42.9 & 42.8 & 85.6 & 128.3 \\
\hline 22 & 5.9 & 9.8 & 3.9 & 6.1 & 1.4 & 0.1 & 57.6 & 57.6 & 57.6 & 59.0 & 118.0 & 177.0 \\
\hline 23 & 0.9 & 1.4 & 0.6 & 1.3 & 0.1 & 0.0 & 36.0 & 36.0 & 36.0 & 36.0 & 72.0 & 108.0 \\
\hline 24 & 0.5 & 0.8 & 0.3 & 0.5 & 0.0 & 0.0 & 44.0 & 44.0 & 44.0 & 43.8 & 87.5 & 131.2 \\
\hline 25 & 0.4 & 0.7 & 0.3 & 0.5 & 0.0 & 0.0 & 51.5 & 51.5 & 51.5 & 50.4 & 100.7 & 151.1 \\
\hline
\end{tabular}

TABLE 7: Link results of the multiclass CL-SUE model.

\begin{tabular}{|c|c|c|c|c|c|c|}
\hline \multirow{3}{*}{ Link no. } & \multicolumn{6}{|c|}{$\begin{array}{c}\text { The multiclass CL-SUE } \\
\text { Link flow }\end{array}$} \\
\hline & \multicolumn{3}{|c|}{ Time-based } & \multicolumn{3}{|c|}{ Monetary-based } \\
\hline & $m=1$ & $m=2$ & $m=3$ & $m=1$ & $m=2$ & $m=3$ \\
\hline 1 & 243.8 & 406.3 & 162.5 & 222.2 & 392.4 & 159.5 \\
\hline 2 & 116.2 & 193.7 & 77.5 & 137.8 & 207.6 & 80.5 \\
\hline 3 & 153.5 & 255.8 & 102.3 & 133.1 & 221.5 & 97.1 \\
\hline 4 & 86.5 & 144.2 & 57.7 & 106.9 & 178.5 & 62.9 \\
\hline 5 & 213.6 & 355.9 & 142.4 & 187.8 & 246.8 & 99.4 \\
\hline 6 & 183.7 & 306.2 & 122.5 & 167.5 & 367.1 & 157.2 \\
\hline 7 & 213.5 & 355.8 & 142.3 & 197.0 & 252.2 & 99.8 \\
\hline 8 & 31.4 & 52.3 & 20.9 & 31.8 & 5.1 & 0.2 \\
\hline 9 & 157.3 & 262.2 & 104.9 & 104.0 & 207.7 & 95.7 \\
\hline 10 & 56.1 & 93.6 & 37.4 & 92.9 & 44.5 & 4.1 \\
\hline 11 & 242.3 & 403.8 & 161.5 & 200.9 & 404.8 & 175.6 \\
\hline 12 & 54.8 & 91.4 & 36.6 & 79.0 & 83.7 & 23.1 \\
\hline 13 & 215.4 & 359.0 & 143.6 & 195.4 & 461.9 & 197.1 \\
\hline 14 & 86.2 & 143.6 & 57.5 & 110.9 & 88.8 & 23.2 \\
\hline 15 & 57.7 & 96.2 & 38.5 & 99.2 & 95.2 & 24.4 \\
\hline 16 & 84.6 & 141.0 & 56.4 & 104.6 & 38.1 & 3.0 \\
\hline 17 & 31.3 & 52.1 & 20.9 & 41.0 & 10.5 & 0.6 \\
\hline 18 & 85.0 & 141.6 & 56.6 & 96.8 & 197.1 & 79.9 \\
\hline 19 & 215.4 & 359.0 & 143.6 & 195.4 & 461.9 & 197.1 \\
\hline
\end{tabular}

Bound (48) becomes $\rho_{\text {CL-SUE }}^{c}=1.1691 \leq 1+\widetilde{\gamma}(\varphi, \beta)+\bar{k} / \theta \widetilde{c} / 1-$ $\bar{\gamma}(\varphi, \beta)=1.4774$ according to Theorem 2. Bound (61) becomes $\quad \rho_{\mathrm{CL}-\mathrm{SUE} / \mathrm{CL}-\mathrm{SSO}}^{t}=\quad 232.6662 \leq \gamma(\varphi) T_{\mathrm{CL}-\mathrm{SUE}}^{t}=$
46179.5710 according to Theorem 3. Bound (67) becomes $\rho_{\mathrm{CL}-\mathrm{SUE} / \mathrm{CL}-\mathrm{SSO}}^{c}=610.8827 \leq \quad \bar{\gamma}(\varphi, \beta) \quad T_{\mathrm{CL}-\mathrm{SUE}}^{c}{ }^{+}$ $\widetilde{\gamma}(\varphi, \beta) T_{\mathrm{CL}-\mathrm{SSO}}^{c}=52418.1288$ according to Theorem 4 . 
TABLE 8: Link results of the multiclass SO and CL-SSO models.

\begin{tabular}{|c|c|c|c|c|c|c|c|c|c|c|c|c|}
\hline \multirow{4}{*}{ Link no. } & \multicolumn{6}{|c|}{ The multiclass SO } & \multicolumn{6}{|c|}{ The multiclass CL-SSO } \\
\hline & \multicolumn{6}{|c|}{ Link flow } & \multicolumn{6}{|c|}{ Link flow } \\
\hline & \multicolumn{3}{|c|}{ Time-based } & \multicolumn{3}{|c|}{ Monetary-based } & \multicolumn{3}{|c|}{ Time-based } & \multicolumn{3}{|c|}{ Monetary-based } \\
\hline & $m=1$ & $m=2$ & $m=3$ & $m=1$ & $m=2$ & $m=3$ & $m=1$ & $m=2$ & $m=3$ & $m=1$ & $m=2$ & $m=3$ \\
\hline 1 & 287.1 & 478.6 & 191.4 & 282.6 & 471.0 & 188.4 & 255.4 & 425.7 & 170.3 & 255.4 & 428.8 & 171.8 \\
\hline 2 & 72.9 & 121.4 & 48.6 & 77.4 & 129.0 & 51.6 & 104.6 & 174.3 & 69.7 & 104.7 & 171.2 & 68.2 \\
\hline 3 & 70.7 & 117.9 & 47.1 & 87.0 & 145.1 & 58.0 & 111.7 & 186.2 & 74.5 & 111.9 & 171.7 & 68.0 \\
\hline 4 & 169.3 & 282.1 & 112.9 & 153.0 & 254.9 & 102.0 & 128.3 & 213.8 & 85.5 & 128.1 & 228.3 & 92.0 \\
\hline 5 & 212.1 & 353.6 & 141.4 & 238.0 & 396.7 & 158.7 & 217.3 & 362.1 & 144.8 & 217.6 & 365.4 & 146.5 \\
\hline 6 & 145.7 & 242.9 & 97.1 & 131.6 & 219.4 & 87.7 & 149.9 & 249.8 & 99.9 & 149.7 & 235.1 & 93.3 \\
\hline 7 & 212.1 & 353.6 & 141.4 & 238.0 & 396.7 & 158.7 & 239.3 & 398.8 & 159.5 & 239.6 & 401.0 & 160.0 \\
\hline 8 & 0.0 & 0.0 & 0.0 & 0.0 & 0.0 & 0.0 & 8.5 & 14.1 & 5.7 & 8.5 & 6.3 & 2.4 \\
\hline 9 & 117.9 & 196.4 & 78.6 & 123.9 & 206.5 & 82.6 & 106.7 & 177.9 & 71.2 & 106.7 & 179.9 & 71.9 \\
\hline 10 & 94.3 & 157.1 & 62.9 & 114.1 & 190.2 & 76.1 & 132.5 & 220.9 & 88.3 & 132.9 & 221.1 & 88.1 \\
\hline 11 & 190.7 & 317.9 & 127.1 & 201.3 & 335.5 & 134.2 & 180.9 & 301.4 & 120.6 & 180.8 & 309.2 & 124.2 \\
\hline 12 & 109.3 & 182.1 & 72.9 & 98.7 & 164.5 & 65.8 & 98.1 & 163.5 & 65.4 & 98.2 & 152.5 & 60.8 \\
\hline 13 & 205.7 & 342.9 & 137.1 & 185.9 & 309.8 & 123.9 & 180.1 & 300.1 & 120.0 & 179.7 & 310.9 & 124.5 \\
\hline 14 & 109.3 & 182.1 & 72.9 & 98.7 & 164.5 & 65.8 & 106.6 & 177.7 & 71.1 & 106.7 & 158.8 & 63.2 \\
\hline 15 & 109.3 & 182.1 & 72.9 & 98.7 & 164.5 & 65.8 & 119.2 & 198.6 & 79.4 & 119.2 & 190.8 & 75.8 \\
\hline 16 & 94.3 & 157.1 & 62.9 & 114.1 & 190.2 & 76.1 & 120.0 & 199.9 & 80.0 & 120.4 & 189.1 & 75.5 \\
\hline 17 & 0.0 & 0.0 & 0.0 & 0.0 & 0.0 & 0.0 & 30.5 & 50.8 & 20.3 & 30.6 & 41.9 & 15.9 \\
\hline 18 & 72.9 & 121.4 & 48.6 & 77.4 & 129.0 & 51.6 & 74.1 & 123.5 & 49.4 & 74.1 & 129.4 & 52.3 \\
\hline 19 & 205.7 & 342.9 & 137.1 & 185.9 & 309.8 & 123.9 & 180.1 & 300.1 & 120.0 & 179.7 & 310.9 & 124.5 \\
\hline
\end{tabular}

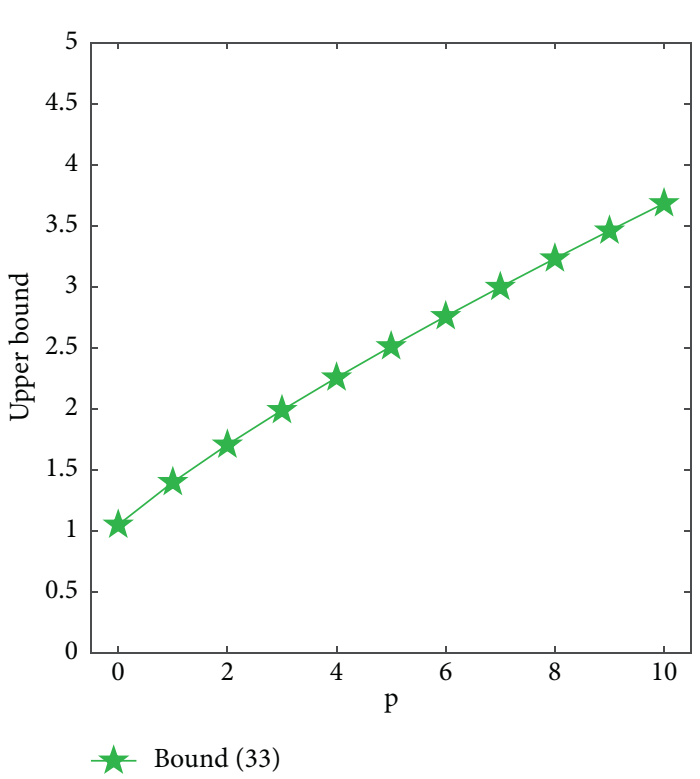

(a)

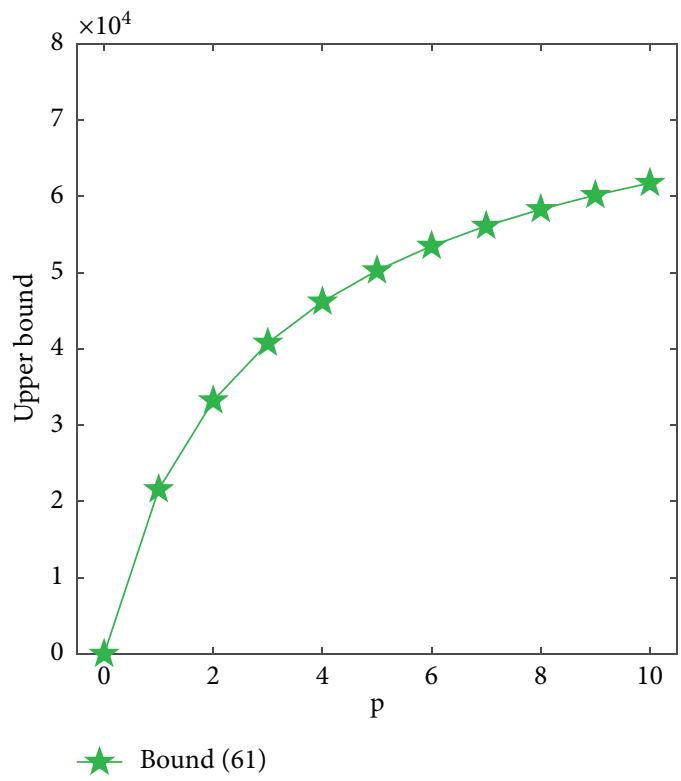

(b)

Figure 3: Bound (33) and bound (61) as a function of $p$.

In summary, the numerical results show that Theorems 1-4 are correct and valid.

Next, we will select three representative parameters for sensitivity analysis. The sensitivities of the upper bounds with respect to parameters $\gamma(\varphi), \bar{k}$, and $\theta$ are addressed in the following. According to the above results, we can obtain $\gamma(\varphi)=0.535, \quad \bar{\gamma}(\varphi, \beta)=0.3058, \quad \tilde{\gamma}(\varphi, \beta)=0, \quad \bar{k}=0.9397$, $\theta=0.5, \quad \bar{c}=38.8391, \quad \tilde{c}=73.3117, \quad$ and $\quad T_{\mathrm{CL}-S U E}^{t}=$ 86316.9550. Since (33) and (68), it is easy to know that bound (33) as a function of $p$ and the function expression is $\rho_{\mathrm{CL}-\mathrm{SUE}}^{t}(p)=1.0484\left(1-(p /(p+1))(1 /(p+1))^{1 / p}\right)^{-1}$. By the same way, we can obtain the other five function expressions: (1) bound (33) as a function of $\bar{k}$ and the function expression is $\rho_{\mathrm{CL}-\mathrm{SUE}}^{t}(\bar{k})=1 / 0.465(1+\bar{k} / 19.4196)$; (2) bound 


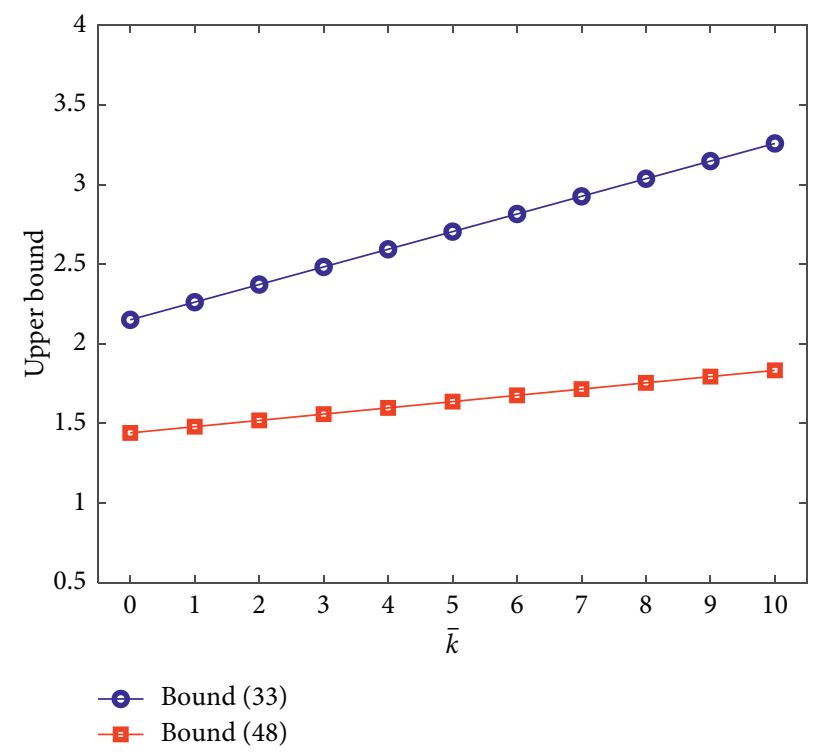

Figure 4: Bound (33) and bound (48) as a function of $\bar{k}$.

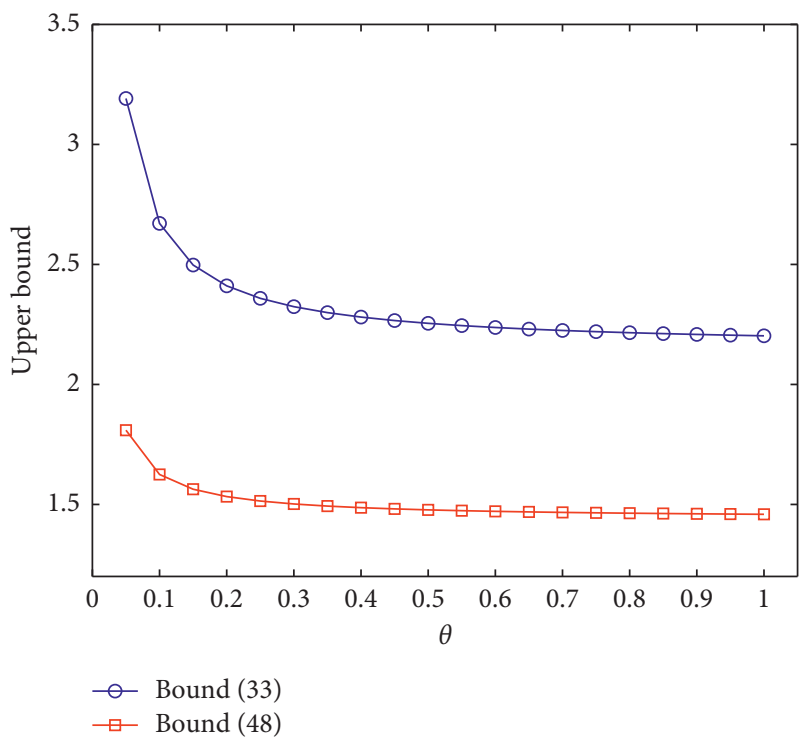

Figure 5: Bound (33) and bound (48) as a function of $\theta$.

(33) as a function of $\theta$ and the function expression is $\rho_{\text {CL-SUE }}^{t}(\theta)=1 / 0.465(1+0.0242 / \theta)$; (3) bound (48) as a function of $\bar{k}$ and the function expression is $\rho_{\text {CL-SUE }}^{c}(\bar{k})=1 / 0.6942(1+\bar{k} / 36.6559) ;(4)$ bound (48) as a function of $\theta$ and the function expression is $\rho_{\text {CL-SUE }}^{c}(\theta)=1 / 0.6942(1+0.0128 / \theta)$; and (5) bound (61) as a function of $p$ and the function expression is $\rho_{\text {CL-SUE/CL-SSO }}^{t}(p)=86317(p /(p+1))(1 /(p+1))^{1 / p}$. The following figures present the sensitivities of upper bounds subject to parameters $p, \bar{k}$, and $\theta$ (see Figures $3-5$ ).

Figure 3 shows both bound (33) and bound (61) increase with $p$; Figure 4 shows both bound (33) and bound (48) increase with $\bar{k}$, and bound (48) is always smaller than bound (33); and Figure 5 shows both bound (33) and bound (48) decrease with $\theta$, and bound (48) is always smaller than bound (33). Therefore, our sensitivity analysis results are consistent with the conclusions given in Section 5, which means that the conclusions given in Section 5 are valid.

\section{Conclusions}

We have derived four inefficiency bounds of the multiclass CL-SUE by making full use of equivalent VI formulations and provide some main conclusions as follows.

When comparing multiclass CL-SUE with multiclass SO, the upper bound under time-based criterion depends on the class of link time functions, the network complexity (including the commonality factor reflecting path overlapping and the number of feasible paths), the perception error of travel time, and the total demand. The upper bound will be underestimated in a realistic transportation network, if the commonality factor is not considered. By contrast, besides the factors mentioned above, the upper bound under monetary-based criterion is dependent upon the VOT of user classes.

When comparing multiclass CL-SUE with multiclass CL-SSO, the upper bound under monetary-based criterion depends on the class of link time functions and the VOT of user classes. However, the upper bound under time-based criterion only depends on the class of link time functions. Moreover, our research results suggest that the upper bound will increase when the degree of traffic congestion goes up. The upper bound is usually not tight in most cases because traffic congestion is inevitable, which means that there is more space to improve the congestion in the traffic network.

Furthermore, the effects of various parameters on the bounds have been further studied, especially the commonality factor. The results of the above discussions are shown in Table 2.

Our study can be further extended in two ways: (1) to explore more accurate upper bounds when more detailed network information is known and (2) to investigate the 
inefficiency of the multiclass CL-SUE with elastic demand under different decision criteria.

\section{Abbreviations}

CL-SUE: C-logit stochastic user equilibrium

VOT: Values of time

VI: $\quad$ Variational inequality

CL-SSO: C-Logit stochastic system optimum

UE: $\quad$ User equilibrium

SO: $\quad$ System optimum

BRUE: Boundedly rational user equilibrium

IID: $\quad$ Independent and identical distributed

SUE: $\quad$ Stochastic user equilibrium

GNL-SUE: Generalized nested logit SUE

CNL-SUE: Cross-nested logit SUE

PCL-SUE: Paired combinatorial logit SUE

MP: $\quad$ Mathematical programming

POA: $\quad$ Price of anarchy

SSO: $\quad$ Stochastic system optimum

TSTT: Total system travel time

TSTC: $\quad$ Total system travel cost

ATIS: $\quad$ Advanced traveler information systems

TPTT: $\quad$ Total perceived travel time

OD: $\quad$ Origin-destination

TPTC: $\quad$ Total perceived travel cost

BPR: $\quad$ Bureau of public road.

\section{Appendix}

Now, we consider the simple network consisting of 2 nodes, $n$ paths, and one OD pair with a demand $d$ and with provided path flows $f_{r}, r \in\{1,2, \cdots, n\}$. Let $u_{1}, u_{2}, \cdots, u_{n}$ be a user's perceived values of the path travel time and $f\left(u_{1}, u_{2}, \cdots, u_{n}\right)$ denote the probability density function of the user's perceived travel time. The mean perceived path travel time $c_{1}, c_{2}, \cdots, c_{n}$ are functions of the path flows $f_{r}$. All users need to be assigned to paths to minimize their TPTT, by dividing the whole space of perceived travel time $u=$ $\left(u_{1}, u_{2}, \cdots, u_{n}\right)$ into mutually exclusive and exhaustive regions $\left\{B_{r}\right\}$ in an optimal manner. The region $B_{r}$ is that within which $u_{r}-h_{r}<u_{k}-h_{k}, k \in\{1,2, \cdots, n\}$, where $h_{r}$ should be given so that the proportion assigned by the above process to path $r$ is $f_{r} / d$. Hence,

$$
\int_{B_{r}} f\left(u_{1}, u_{2}, \cdots, u_{n}\right) d u_{1} d u_{2} \cdots d u_{n}=p_{r}=\frac{f_{r}}{d} .
$$

With this assignment, the TPTT of the network with one OD pair is

$$
F\left(f_{1}, f_{2}, \cdots, f_{r}\right)=d \sum_{r} \int_{B_{r}} u_{r} f\left(u_{1}, u_{2}, \cdots, u_{n}\right) d u_{1} d u_{2} \cdots d u_{n} .
$$

We suppose that only the means $c_{1}, c_{2}, \cdots, c_{n}$ are influenced by path flows $f_{r}$ and the variances and covariances remain constant. Hence, the density function satisfies the condition below.

$$
\begin{aligned}
& f\left(u_{1}+h_{1}, u_{2}+h_{2}, \cdots, u_{n}+h_{n} ; c_{1}, c_{2}, \cdots, c_{n}\right) \\
& \quad=f\left(u_{1}, u_{2}, \cdots, u_{n} ; c_{1}-h_{1}, c_{2}-h_{2}, \cdots, c_{n}-h_{n}\right) .
\end{aligned}
$$

Setting $w_{r}=u_{r}-h_{r}$ and denoting by $Q_{r}$ the set of perceived path travel time for which path $r$ is the optimum, (A2) can be rewritten as

$$
\begin{aligned}
F\left(f_{1}, f_{2}, \cdots, f_{r}\right) & =d \sum_{r} \int_{Q_{r}}\left(w_{r}+h_{r}\right) f\left(w_{1}+h_{1}, w_{2}+h_{2}, \cdots, w_{n}+h_{n} ; c_{1}, c_{2}, \cdots, c_{n}\right) d w_{1} d w_{2} \cdots d w_{n}, \\
& =d \sum_{r} \int_{Q_{r}}\left(w_{r}+h_{r}\right) f\left(w_{1}, w_{2}, \cdots, w_{n} ; c_{1}-h_{1}, c_{2}-h_{2}, \cdots, c_{n}-h_{n}\right) d w_{1} d w_{2} \cdots d w_{n}, \\
& =d \sum_{r} \int_{Q_{r}} w_{r} f\left(w_{1}, w_{2}, \cdots, w_{n} ; c_{1}-h_{1}, c_{2}-h_{2}, \cdots, c_{n}-h_{n}\right) d w_{1} d w_{2} \cdots d w_{n}+d \sum_{r} h_{r} p_{r}, \\
& =d S\left(c_{1}-h_{1}, c_{2}-h_{2}, \cdots, c_{n}-h_{n}\right)+\sum_{r} h_{r} f_{r},
\end{aligned}
$$

where $S$ is the composite travel time. In the logit case, $S$ is provided by a specific "logsum" expression:

$$
\begin{aligned}
& S\left(c_{1}-h_{1}, c_{2}-h_{2}, \cdots, c_{n}-h_{n}\right) \\
& \quad=-\frac{1}{\theta} \log \left(\sum_{r} \exp \left(-\theta\left(c_{r}-h_{r}\right)\right)\right) .
\end{aligned}
$$

Suppose that we have a multiple OD pair transportation network with multiple classes of users. The demand of user class $m$ between OD pair $w \in W$ is denoted by $d_{w}^{m}$. The flow of user class $m$ on path $r \in R_{w}, w \in W$ is expressed by $f_{r w}^{m}$. Let $c_{r w}^{m}$ denote the mean perceived travel time of user class $m$ on path $r \in R_{w}, w \in W$. Therefore, the TPTT is given by

$$
\begin{aligned}
F(f)= & \sum_{w \in W} \sum_{m=1}^{M} d_{w}^{m} S_{w}^{m}\left(c_{w}^{m}-h_{w}^{m}\right) \\
& +\sum_{w \in W} \sum_{r \in R_{w}} \sum_{m=1}^{M} h_{r w}^{m} f_{r w}^{m} .
\end{aligned}
$$

Here, we have 


$$
S_{w}^{m}\left(c_{w}^{m}-h_{w}^{m}\right)=-\frac{1}{\theta} \log \left(\sum_{r} \exp \left(-\theta\left(c_{r w}^{m}-h_{r w}^{m}\right)\right)\right)
$$

In the C-Logit case, we suppose that $c_{r w}^{\prime m}=c_{r w}^{m}+c f_{r w}$ is the mean perceived travel time, where $c_{r w}^{m}$ is the actual travel time of user class $m$ on path $r \in R_{w}, w \in W$ and $c f_{r w}$ is a commonality factor of path $r \in R_{w}, w \in W$. The path choice probability for user class $m$ is given by

$$
p_{r w}^{m}=\frac{\exp \left(-\theta\left(c_{r w}^{\prime m}-h_{r w}^{m}\right)\right)}{\sum_{l \in R_{w}} \exp \left(-\theta\left(c_{r w}^{\prime m}-h_{r w}^{m}\right)\right)}, r \in R_{w}, w \in W, m=1,2, \cdots, M .
$$

Hence, we can obtain the values of $h_{r w}^{m}$ and $h_{l w}^{m}$ from

$$
\ln \frac{f_{r w}^{m}}{f_{l w}^{m}}=-\theta\left({c^{\prime}}_{r w}^{m}-h_{r w}^{m}-c_{l w}^{\prime m}+h_{l w}^{m}\right)
$$

so that

$$
h_{r w}^{m}-h_{l w}^{m}=c_{r w}^{\prime m}-c_{l w}^{\prime m}+\frac{1}{\theta} \ln \frac{f_{r w}^{m}}{f_{l w}^{m}}
$$

Here, we can set any one of the $h_{r w}^{m}$ to zero. Setting $h_{1 w}^{m}=0$, the value of $h_{r w}^{m}$ is given by

$$
h_{r w}^{m}=c_{r w}^{\prime m}-c_{1 w}^{\prime m}+\frac{1}{\theta} \ln \frac{f_{r w}^{m}}{f_{1 w}^{m}} .
$$

For any one OD pair $w$, we can have the following objective function:

$$
\begin{aligned}
F\left(f_{w}^{m}\right)= & -\frac{1}{\theta} \sum_{m=1}^{M} d_{w}^{m} \ln \left(\sum_{r} \exp \left(-\theta\left(c_{r w}^{\prime m}-h_{r w}^{m}\right)\right)\right) \\
& +\sum_{r \neq 1} \sum_{m=1}^{M} f_{r w}^{m}\left(c_{r w}^{\prime m}-c_{1 w}^{\prime m}+\frac{1}{\theta} \ln \frac{f_{r w}^{m}}{f_{1 w}^{m}}\right) .
\end{aligned}
$$

Substituting (A11) into (A12) and summing all OD pairs, the objective function for the multiclass CL-SSO is given as follows:

$$
\begin{aligned}
F(f) & =-\frac{1}{\theta} \sum_{w \in W} \sum_{m=1}^{M} d_{w}^{m} \ln \left(\sum_{r} \exp \left(-\theta\left(c_{r w}^{\prime m}-h_{r w}^{m}\right)\right)+\sum_{w \in W} \sum_{r \in R_{w}, r \neq 1} \sum_{m=1}^{M} f_{r w}^{m}\left(c_{r w}^{\prime m}-c_{1 w}^{\prime m}+\frac{1}{\theta} \ln \frac{f_{r w}^{m}}{f_{1 w}^{m}}\right)\right. \\
& =\sum_{w \in W} \sum_{r \in R_{w}} \sum_{m=1}^{M} f_{r w}^{m} c_{r w}^{\prime m}+\frac{1}{\theta} \sum_{w \in W} \sum_{r \in R_{w}} \sum_{m=1}^{M} f_{r w}^{m} \ln f_{r w}^{m}-\frac{1}{\theta} \sum_{w \in W} \sum_{m=1}^{M} d_{w}^{m} \ln \left(\sum_{r} \exp \left(-\theta\left(c_{1 w}^{\prime m}-\frac{1}{\theta} \ln \frac{f_{r w}^{m}}{f_{1 w}^{m}}\right)\right)\right) \\
& =\sum_{w \in W} \sum_{r \in R_{w}} \sum_{m=1}^{M} f_{r w}^{m} c_{r w}^{\prime m}+\frac{1}{\theta} \sum_{w \in W} \sum_{r \in R_{w}} \sum_{m=1}^{M} f_{r w}^{m} \ln f_{r w}^{m}-\frac{1}{\theta} \sum_{w \in W} \sum_{m=1}^{M} d_{w}^{m} \ln d_{w}^{m} .
\end{aligned}
$$

Substituting ${c_{r w}^{\prime m}}_{r w}^{m}=c_{r w}^{m}+c f_{r w}$ into (A13), we can obtain

$$
\begin{aligned}
F(f)= & \sum_{w \in W} \sum_{r \in R_{w}} \sum_{m=1}^{M} f_{r w}^{m}\left(c_{r w}^{m}(f)+c f_{r w}\right) \\
& +\frac{1}{\theta} \sum_{w \in W} \sum_{r \in R_{w}} \sum_{m=1}^{M} f_{r w}^{m} \ln f_{r w}^{m}-\frac{1}{\theta} \sum_{w \in W} \sum_{m=1}^{M} d_{w}^{m} \ln d_{w}^{m} .
\end{aligned}
$$

\section{Data Availability}

The data used to support the findings of this study are included within the article.

\section{Conflicts of Interest}

The authors declare that they have no conflicts of interest.

\section{Acknowledgments}

This research was funded by the National Social Science Foundation of China (Grant no. 16BJL121), Chongqing
Municipal Education Commission Science and Technology Research Project (Grant no. KJ1705148), and Chongqing Jiaotong University Research Start-up Funding Project (Grant no. 17JDKJC-A002).

\section{References}

[1] WardropCorrespondence, "Some theoretical aspects of road traffic research," Proceedings - Institution of Civil Engineers, vol. 1, no. 5, pp. 767-768, 1952.

[2] Mahmassani and Chang, "On boundedly rational user equilibrium in transportation systems," Transportation Science, vol. 21, no. 2, pp. 89-99, 1987.

[3] Y. Lou, Y. Yin, and S. Lawphongpanich, "Robust congestion pricing under boundedly rational user equilibrium," Transportation Research Part B: Methodological, vol. 44, no. 1, pp. 15-28, 2010.

[4] D. Xuan, H. X. Liu, J. S. Pang, and X. Ban, "Boundedly rational user equilibria (BRUE): mathematical formulation and solution sets," Procedia-Social and Behavioral Sciences, vol. 80, pp. 231-248, 2013.

[5] H. Ramazani, Y. Shafahi, and S. E. Seyedabrishami, "A fuzzy traffic assignment algorithm based on driver perceived travel 
time of network links," Scientia Iranica, vol. 18, no. 2, pp. 190-197, 2011.

[6] M. Miralinaghi, Y. Shafahi, and R. S. Anbarani, "A fuzzy network assignment model based on user equilibrium condition," Scientia Iranica, vol. 22, no. 6, pp. 2012-2023, 2015.

[7] M. Miralinaghi, Y. Lou, Y. T. Hsu, R. Shabanpour, and Y. Shafahi, "Multiclass fuzzy user equilibrium with endogenous membership functions and risk-taking behaviors," Journal of Advanced Transportation, vol. 50, no. 8, pp. 1716-1734, 2016.

[8] R. D. Connors and A. Sumalee, "A network equilibrium model with travellers' perception of stochastic travel times," Transportation Research Part B: Methodological, vol. 43, no. 6, pp. 614-624, 2009.

[9] S. Gao, E. Frejinger, and M. Ben-Akiva, "Adaptive route choices in risky traffic networks: a prospect theory approach," Transportation Research Part C: Emerging Technologies, vol. 18, no. 5, pp. 727-740, 2010.

[10] H. Xu, Y. Lou, Y. Yin, and J. Zhou, "A prospect-based user equilibrium model with endogenous reference points and its application in congestion pricing," Transportation Research Part B: Methodological, vol. 45, no. 2, pp. 311-328, 2011.

[11] Y. Sheffi, Urban Transportation Networks: Equilibrium Analysis with Mathematical Programming Methods, pp. 320322, Prentice-Hall, Englewood Cliffs, Upper Saddle River, NJ, USA, 1985.

[12] C. Fisk, "Some developments in equilibrium traffic assignment," Transportation Research Part B: Methodological, vol. 14, no. 3, pp. 243-255, 1980.

[13] E. Cascetta, A. Nuzzolo, F. Russo, and A. Vitetta, "A modified logit route choice model overcoming path overlapping problems: specification and some calibration results for interurban networks," in Proceedings of the 13th International Symposium on Transportation and Traffic Theory, pp. 697-711, Lyon, France, 1996.

[14] Z. Zhou, A. Chen, and S. Bekhor, "C-logit stochastic user equilibrium model: formulations and solution algorithm," Transportmetrica, vol. 8, no. 1, pp. 17-41, 2012.

[15] B. Q. Liu, Y. H. Zhang, and W. Du, "A simplified C-Logit stochastic user equilibrium model on bimodal transportation network," Mathematical Problems in Engineering, vol. 2020, Article ID 3702965, 8 pages, 2020.

[16] X. Xu and A. Chen, "C-logit stochastic user equilibrium model with elastic demand," Transportation Planning and Technology, vol. 36, no. 5, pp. 463-478, 2013.

[17] S. Bekhor and Prashker, "Stochastic user equilibrium formulation for generalized nested logit model," Transportation Research Record: Journal of the Transportation Research Board, vol. 1752, no. 1, pp. 84-90, 2001.

[18] A. Papola, "Some developments on the cross-nested logit model," Transportation Research Part B: Methodological, vol. 38, no. 9, pp. 833-851, 2004.

[19] S. Bekhor, T. Toledo, and L. Reznikova, "A path-based algorithm for the cross-nested logit stochastic user equilibrium," Computer-Aided Civil and Infrastructure Engineering, vol. 24, no. 1, pp. 15-25, 2010.

[20] A. Chen, P. Kasikitwiwat, and Z. Ji, "Solving the overlapping problem in route choice with paired combinatorial logit model," Transportation Research Record: Journal of the Transportation Research Board, vol. 1857, no. 1, pp. 65-73, 2003.

[21] A. Chen, S. Pravinvongvuth, X. Xu, S. Ryu, and P. Chootinan, "Examining the scaling effect and overlapping problem in logit-based stochastic user equilibrium models,"
Transportation Research Part A: Policy and Practice, vol. 46, no. 8, pp. 1343-1358, 2012.

[22] P. H. L. Bovy, S. Bekhor, and C. G. Prato, "The factor of revisited path size alternative derivation," Transportation Research Record, vol. 2076, pp. 132-140, 2008.

[23] E. Koutsoupias and C. Papadimitriou, "Worst-case equilibria, s," in Proceedings of the 16th Annual Symposium on Theoretical Aspects of Computer Science (STACS), pp. 404-413, Trier, Germany, March 1999.

[24] T. Roughgarden and É. Tardos, "How bad is selfish routing?" Journal of the ACM, vol. 49, no. 2, pp. 236-259, 2002.

[25] T. Roughgarden, "The price of anarchy is independent of the network topology," Journal of Computer and System Sciences, vol. 67, no. 2, pp. 341-364, 2003.

[26] T. Roughgarden and É. Tardos, "Bounding the inefficiency of equilibria in nonatomic congestion games," Games and Economic Behavior, vol. 47, no. 2, pp. 389-403, 2004.

[27] T. Roughgarden, Selfish Routing and the Price of Anarchy, The MIT Press, Cambridge, Massachusetts, 2005.

[28] C. K. Chau and K. M. Sim, "The price of anarchy for nonatomic congestion games with symmetric cost maps and elastic demands," Operations Research Letters, vol. 31, no. 5, pp. 327-334, 2003.

[29] J. R. Correa, A. S. Schulz, and N. E. Stier-Moses, "Selfish routing in capacitated networks," Mathematics of Operations Research, vol. 29, no. 4, pp. 961-976, 2004.

[30] H. Yang, D. Han, and H. K. Lo, "Efficiency of atomic splittable selfish routing with polynomial cost functions," Networks and Spatial Economics, vol. 8, no. 4, pp. 443-451, 2008.

[31] H. Yang, W. Xu, and B. Heydecker, "Bounding the efficiency of road pricing," Transportation Research Part E: Logistics and Transportation Review, vol. 46, no. 1, pp. 90-108, 2010.

[32] T.-L. Liu, J. Chen, and H.-J. Huang, "Existence and efficiency of oligopoly equilibrium under toll and capacity competition," Transportation Research Part E: Logistics and Transportation Review, vol. 47, no. 6, pp. 908-919, 2011.

[33] Z. Feng, Z. Gao, and H. Sun, "Bounding the inefficiency of atomic splittable selfish traffic equilibria with elastic demands," Transportation Research Part E: Logistics and Transportation Review, vol. 63, pp. 31-43, 2014.

[34] D. Han, H. K. Lo, J. Sun, and H. Yang, "The toll effect on price of anarchy when costs are nonlinear and asymmetric," European Journal of Operational Research, vol. 186, no. 1, pp. 300-316, 2008.

[35] S. J. O'Hare, R. D. Connors, and D. P. Watling, "Mechanisms that govern how the Price of Anarchy varies with travel demand," Transportation Research Part B: Methodological, vol. 84, pp. 55-80, 2016.

[36] M. L. Mouronte-Lo'pez, "Modeling the public transport networks: a study of their efficiency," Complexity, vol. 2021, Article ID 3280777, 19 pages, 2021.

[37] X. Guo, H. Yang, and T.-L. Liu, "Bounding the inefficiency of logit-based stochastic user equilibrium," European Journal of Operational Research, vol. 201, no. 2, pp. 463-469, 2010.

[38] X. Yu, H. Huang, and T. Liu, "Efficiency loss of the multiclass stochastic traffic equilibrium assignment with fixed demand," Journal of Transportation Systems Engineering and Information Technology, vol. 9, no. 4, pp. 83-89, 2009.

[39] G. Yong, H. Huang, T. Liu, and Y. Xu, "Bounding the inefficiency of the C-Logit stochastic user equilibrium assignment," Journal of Systems Science and Complexity, vol. 29, no. 6, pp. 1629-1649, 2016.

[40] M. Zeng and Y. Wang, "Upper bounds on inefficiency of taxed stackelberg network with cross- nested logit assignment," The 
Open Cybernetics \& Systemics Journal, vol. 8, no. 1, pp. 1091-1098, 2014.

[41] Guo and H. Yang, "User heterogeneity and bi-criteria system optimum," Transportation Research Part B: Methodological, vol. 43, no. 4, pp. 379-390, 2009.

[42] X.-J. Yu and L.-L. Wang, "On efficiency loss of multiclass traffic equilibrium assignment with elastic demand," Procedia - Social and Behavioral Sciences, vol. 138, pp. 368-377, 2014.

[43] D. R. Han and H. Yang, "The multi-class, multi-criterion traffic equilibrium and the efficiency of congestion pricing," Transportation Research Part E: Logistics and Transportation Review, vol. 44, no. 5, pp. 753-773, 2008.

[44] H.-J. Huang, T.-L. Liu, X. Guo, and H. Yang, "Inefficiency of logit-based stochastic user equilibrium in a traffic network under ATIS," Networks and Spatial Economics, vol. 11, no. 2, pp. 255-269, 2011.

[45] X. J. Yu and H. J. Huang, "Efficiency loss of mixed equilibrium behaviors with polynomial cost functions," Promet - Traffic \& Transportation, vol. 22, no. 5, pp. 325-331, 2010.

[46] X.-J. Yu and C.-H. Fang, "Efficiency loss of mixed equilibrium associated with altruistic users and logit-based stochastic users in transportation network," Promet - Traffic \& Transportation, vol. 26, no. 1, pp. 45-51, 2014.

[47] X. J. Yu and J. C. Long, "A mixed traffic assignment problem and its upper bound of efficiency loss," Journal of Applied Mathematics, vol. 2014, Article ID 290972, 7 pages, 2014.

[48] M. Maher, K. Stewart, and A. Rosa, "Stochastic social optimum traffic assignment," Transportation Research Part B: Methodological, vol. 39, no. 8, pp. 753-767, 2005.

[49] H. Yang, "System optimum, stochastic user equilibrium, and optimal link tolls," Transportation Science, vol. 33, no. 4, pp. 354-360, 1999.

[50] S. Nguyen and C. Dupuis, "An efficient method for computing traffic equilibria in networks with asymmetric transportation costs," Transportation Science, vol. 18, no. 2, pp. 185-202, 1984. 